\begin{tabular}{|l|c|c|c|c|}
\hline $\begin{array}{l}\text { Cuadernos de Investigación Geográfica } \\
\text { Geographical Research Letters }\end{array}$ & 2019 & N $^{\circ} 45(1)$ & pp. 167-193 & eISSN 1697-9540 \\
\hline
\end{tabular}

\title{
ESCENARIOS, TEMPO E INDICADORES PALEOAMBIENTALES PARA LA IDENTIFICACIÓN DEL ANTROPOCENO EN EL PAISAJE VEGETAL DEL PIRINEO CENTRAL (NE IBERIA)
}

\author{
P. GONZÁLEZ-SAMPÉRIZ ${ }^{1 *}$, L. MONTES ${ }^{2}$, J. ARANBARRI ${ }^{3}$, M. LEUNDA $^{1}$, \\ R. DOMINGO ${ }^{2}$, R. LABORDA ${ }^{2}$, Y. SANJUÁN ${ }^{1}$, G. GIL-ROMERA ${ }^{1,4}$, T. LASANTA ${ }^{1}$, \\ J.M. GARCÍA-RUIZ ${ }^{1}$
}

\begin{abstract}
${ }^{1}$ Dpto. Procesos Geoambientales y Cambio Global, Instituto Pirenaico de Ecología-CSIC, Av/ Montañana 1005, 50059 Zaragoza (Spain). ${ }^{2}$ Dpto. Ciencias de la Antigüedad (Área de Prehistoria), Universidad de Zaragoza, C/ Pedro Cerbuna 12, 50009 Zaragoza (Spain).

${ }^{3}$ Dpto. Geografía, Prehistoria y Arqueología, Universidad del Pais Vasco (UPV-EHU), C/ Tomás y Valiente s/n, 01006 Vitoria-Gasteiz (Spain).

${ }^{4}$ Dep. Geography and Earth Sciences, Aberystwyth University, Penglais Campus, SY23, United Kingdom.
\end{abstract}

RESUMEN. El Antropoceno puede considerarse como el origen de la configuración paisajística actual, y en relación a la vegetación, como el periodo en el que la intervención humana es el agente principal de los cambios que se producen de forma clara, intensa y permanente en el tiempo. En este trabajo se recopilan y comparan datos de secuencias palinológicas y de microcarbones, ocupaciones de yacimientos arqueológicos y dataciones de paleoincendios localizados en el Pirineo Central, además de información documental, exponiendo una reflexión sobre las supuestas evidencias que promulgan una deforestación temprana y el uso del fuego de origen antrópico como señal inequívoca de intervención humana y surgimiento de los paisajes culturales desde hace milenios. En realidad, lo que se constata es una alta diversidad temporal y espacial entre todos los indicios, y sólo a partir de la Baja Edad Media (últimos 700 años, 1300 AD), los indicadores $y$ valores de los mismos ofrecen auténtico consenso sobre el inicio de cambios consistentes y duraderos que marcarían el inicio del Antropoceno.

Scenarios, timing and paleo-environmental indicators for the identification of Anthropocene in the vegetal landscape of the Central Pyrenees (NE Iberia)

ABSTRACT. The Anthropocene is related to the origin of current landscape configuration, and in terms of vegetation, with the consideration of human action as main forcing of clear, intense and permanent changes through time. In this paper we compile and compare data from palynological sequences, microcharcoal records, archaeological sites and radiocarbon dates from palaeofires located in the Central Pyrenees besides historical documents, and we argue that some evidences related to early deforestation processes and use 
of human fire are not so clear than some authors point. Conversely, indicators of unequivocal human use and the origin of cultural landscapes are located only a few centuries and not a few millennia ago. In fact, high temporal and spatial variability is recorded until the Middle Ages (last 700 years, 1300 AD), when a series of consistent and permanent changes suggest the onset of the Anthropocene.

Palabras clave: Antropoceno, alta montaña, polen, fuego, deforestación, uso humano, Pirineo Central.

Key words: Anthropocene, high mountain areas, pollen, fire, deforestation, human use, Central Pyrenees.

Recibido: 31 de julio de 2018

Aceptado: 14 de diciembre de 2018

*Correspondencia: Penélope González Sampériz, Dpto. de Procesos Geoambientales y Cambio Global, Instituto Pirenaico de Ecología, Consejo Superior de Investigaciones Científicas (IPE-CSIC), Apartado 13.034, 50080 Zaragoza (Spain). E-mail: pgonzal@ ipe.csic.es

\section{Introducción: Antropoceno y paisajes culturales}

La identificación, caracterización y consideración del Antropoceno como una nueva era en la historia de nuestro planeta, en la que los humanos ejercemos el papel protagonista en términos de forzamiento de cambio, está invadiendo la literatura de los últimos años (Monastersky, 2015; Zalasiewicz et al., 2017; Waters et al., 2018). Como suele ocurrir cuando una temática deriva en ingente producción científica, existen diversidad de opiniones respecto a su significado y adscripción temporal, e incluso respecto a su consideración como nuevo periodo geológico (Malm y Hornborg, 2014; Finney y Edwards, 2016; Rull, 2018).

Al margen de este contexto dialéctico, en este trabajo se considera el Antropoceno como el surgimiento de la configuración paisajística actual en términos de formaciones vegetales. Esto es, como el origen de lo que actualmente se denominan "paisajes culturales" (Aitchison, 1995). Se entiende por paisaje cultural, aquel que se ha construido a lo largo de siglos o incluso milenios mediante un esfuerzo, necesariamente colectivo, de adaptación de diferentes culturas a la diversidad de la topografía y de los topoclimas, y a la organización espacial de la fertilidad de los suelos y de los flujos hidrológicos (García-Ruiz y Lasanta, 2018). Martínez de Pisón (2009) afirma que los paisajes de montaña son productos históricos y documentan el peso de la cultura sobre el territorio. Algunos autores incluso, afirman que los paisajes de montaña son como un palimpsesto en el que permanecen en mayor o menor medida las diferentes etapas y maneras de aprovechamiento del territorio (Antrop, 2005; Blondel, 2006). De ahí la importancia de la arqueología del paisaje (Fernández Mier et al., 2013; Fernández Mier y Quirós Castillo, 2015) y de los estudios paleoambientales que analizan cambios en la vegetación y en 
las tasas de erosión para identificar cambios en el paisaje, incluso durante el Holoceno Medio y Reciente (Barreiro-Lostres et al., 2017).

Desde hace unos 7600 años, con la llegada del Neolítico a la Península Ibérica (JuanCabanilles y Martí-Oliver, 2002; Utrilla, 2002; Rojo et al., 2012; Martí-Oliver y JuanCabanilles, 2014; Bernabeu et al., 2018), las actividades humanas comenzaron a tener un impacto sin precedentes, y con el tiempo, acabarían modificando de manera notable la dinámica natural del paisaje vegetal. A partir de entonces, la historia de la vegetación se ve influenciada por un nuevo y poderoso actor: el ser humano. El clima seguirá siendo un agente esencial, e incluso dominante en muchos casos y localidades a la hora de determinar la dinámica de la vegetación, pero ya no actuará en solitario, sino reforzado en muchos casos por actividades socio-económicas (Carrión et al., 2010). Granos de polen de cereal y otras plantas cultivadas empiezan a aparecer en las secuencias palinológicas, incluso en las procedentes de registros lacustres y turbosos (Miras et al., 2007; Pèlachs et al., 2009; Ejarque et al., 2010; López-Merino et al., 2010; Aranbarri et al., 2015; Revellés, 2017), completando la información proporcionada por los yacimientos arqueológicos (López-Sáez et al., 2009; Pérez-Díaz et al., 2018), y muy especialmente por la carpología (Zapata et al., 2004; Peña-Chocarro et al., 2005, 2018). Las proporciones de polen arbóreo-arbustivo descienden, o al menos fluctúan intensa y bruscamente, interpretándose tradicionalmente como la expansión intencionada de áreas abiertas con el objetivo de cultivar y/o pastorear (Carrión et al., 2010; López-Merino et al., 2010).

Para unos, éste es el origen del Antropoceno. Para otros, habrá que esperar hasta el comienzo de la metalurgia, la Revolución Industrial o incluso la Gran Aceleración del siglo XX para que el sello humano adquiera ya un carácter global y una intensidad extrema (Zalasiewicz et al., 2017), con consecuencias tanto en la vegetación como en la hidrología, el clima, la política, la economía o la sostenibilidad socio-ambiental (Arias Maldonado, 2018).

En cualquier caso, en lo referente a la vegetación quizá lo más relevante sea determinar qué indicadores, $y$, sobre todo, con qué valores, señalan inequívocamente que los cambios registrados en las secuencias palinológicas son producto de la mano humana, independientemente del cuándo. Pero no es fácil hacerlo en todos los periodos cronológicos ni en todas las localizaciones, ya sea a partir de datos "tradicionales" o utilizando modelos (Roberts et al., 2018).

En este escenario, una de las mejores áreas geográficas para caracterizar cambios de vegetación diversos y su origen es la Cordillera Pirenaica, puesto que aúna una larga tradición cultural y un marco ambiental formado por un complejo mosaico de biodiversidad, ambientes y usos del suelo (García-Ruiz et al., 2015). La combinación de áreas remotas (cotas de mayor altitud y difícil accesibilidad) y valles explotados como lugares de hábitat y actividades económicas diversas desde, al menos, el inicio del Neolítico (Galop, 1999; Rendu, 2003; Gassiot et al., 2012; Galop et al., 2013; Rojo et al., 2013; Clemente et al., 2016; Montes et al., 2016a, 2016b; Rendu et al., 2016; Laborda et al., 2017), permiten plantear cierta reflexión sobre esta temática.

Durante las últimas décadas, la disponibilidad de reconstrucciones palinológicas en el Pirineo ha aumentado significativamente (Ejarque et al., 2010; Pérez-Obiol et al., 
2012; Pérez-Sanz et al., 2013; Garcés-Pastor et al., 2017; González-Sampériz et al., 2017; Leunda et al., 2017, entre otros), pero sigue sin haber consenso ni unas pautas claras en la interpretación de las secuencias disponibles, especialmente en lo referente al uso tradicional de la alta montaña, la deforestación del piso subalpino y la expansión de pastos.

En este trabajo se propone un análisis comparativo de secuencias (Fig. 1), indicadores y proporciones que, en muchos casos, aportan información semejante, si bien el forzamiento o agente de cambio a quien se atribuye el origen, no lo es.

\section{Paradigmas establecidos y contextualización paleoambiental e histórica: Dónde y desde cuándo}

Tradicionalmente, a partir de fuentes documentales se venía considerando que la ocupación y explotación humana de la alta montaña pirenaica de forma sistemática databa de tiempos medievales (Pallaruelo, 1988), aunque sin descartar un posible origen anterior de cronología imprecisa. Desde los años 90, y especialmente en las dos últimas décadas, empezó a extenderse y aceptarse la hipótesis de que fue durante la Prehistoria reciente, o incluso antes (Mesolítico final o Neolítico antiguo, esto es, en torno a hace unos 8000 años), cuando se inició tímidamente el diseño de los anteriormente mencionados "paisajes culturales" (Catalán et al., 2013; Orengo et al., 2014).

En cualquier caso, y exceptuando esta tendencia relativamente reciente a localizar durante el final del Holoceno Temprano e inicios del Holoceno Medio los primeros vestigios de modificación intensa del paisaje vegetal de altitud por parte de los humanos (Rojo et al., 2013; Clemente et al., 2016; Gassiot, 2016), buena parte de la literatura científica establece el Holoceno Reciente (desde hace 4200 años siguiendo la división de Walker et al., 2012) como el comienzo de la intensificación de la presión humana en alta montaña. El objetivo, abrir y mantener espacios abiertos para pastos (Montserrat, 1992; Galop, 2006; Ejarque et al., 2010; Pérez-Díaz et al., 2015), deforestándose el piso subalpino de los Pirineos de forma masiva y persistente desde entonces (García-Ruiz et al., 2015).

A pesar de esta especie de dogma, no todas las evidencias confirman el paradigma (González-Sampériz et al., 2017). Como tampoco lo hace el modus operandi considerado para ese escenario: el uso intencionado del fuego, periódica y recurrentemente, para mantener amplias praderas y espacios abiertos de modo que el bosque no recupere ya su espacio "natural" (Vannière et al., 2001; Gil-Romera et al., 2010; Bal et al., 2011). Los registros de polen, macro y microcarbones publicados hasta el momento en el Pirineo Central no confirman esta idea de manera generalizada (Pérez-Sanz et al., 2011, 2013; Leunda et al., 2017, en revisión) (Fig. 2). Del mismo modo, varios niveles de paleoincendios preservados en depósitos de ladera (Las Blancas, Barranco Pardina, Plandaniz y Araguiés del Puerto) aparecen localizados entre los 1700 y los $2100 \mathrm{~m}$ de altitud, por lo que no se distribuyen por todo el piso subalpino, ni con cronología periódica (Tabla 1). De hecho, han sido datados en periodos aislados como la Edad del Bronce, Hierro, Roma, Tardoantigüedad o Edad Media, pero de forma intermitente y episódica (Fig. 2). 
Tabla 1. Dataciones de niveles de paleoincendios preservados en varios depósitos de ladera en cuatro localizaciones del piso subalpino del Pirineo Central (Huesca): área de Las Blancas, Barranco Pardina en Ordesa, entorno del ibón colmatado de Plandaniz y cabecera de Aragüés del Puerto. Las muestras han sido datadas en los laboratorios de Poznán-Poz (Polonia), Leipzig-MAMS (Alemania) y Livermore-Liv (USA) y calibradas con el programa OxCal v. 4.3 (Bronk Ramsey, 2009).

\begin{tabular}{|c|c|c|c|c|c|}
\hline Sitio/Altitud & Ref. & Muestra & 14C AMS & $\mathbf{\pm}$ & cal BP \\
\hline \multirow{2}{*}{$\left.\begin{array}{c}\text { LAS BLANCAS } \\
(1800-2100\end{array}\right)$} & Poz-56691 & Las Blancas 1 & 1185 & 30 & 1114 \\
\cline { 2 - 6 } & Poz-57803 & Las Blancas 3A & 2505 & 30 & 2598 \\
\cline { 2 - 6 } $\begin{array}{c}\text { BCO. PARDINA } \\
(1790 m)\end{array}$ & Liv-169821 & Las Blancas 40 & 2080 & 30 & 2053 \\
\hline \multirow{4}{*}{$\begin{array}{c}\text { PLANDANIZ } \\
(1722 \text { m) }\end{array}$} & Poz-56695 & Plandaniz A & 2125 & 30 & 2108 \\
\cline { 2 - 6 } & Poz-56696 & Plandaniz B & 2390 & 30 & 2429 \\
\cline { 2 - 6 } & Poz-56697 & Plandaniz C & 2130 & 35 & 2121 \\
\cline { 2 - 6 } & Poz-57818 & Plandaniz E & 1160 & 25 & 1083 \\
\cline { 2 - 6 } & Liv-169734 & Plandaniz 10 & 1690 & 30 & 1601 \\
\hline \multirow{2}{*}{$\begin{array}{c}\text { ARAGÜÉS DEL } \\
\text { PUERTO } \\
(1715 \text { m) }\end{array}$} & Liv-1696735 & Plandaniz 14 & 1095 & 30 & 1005 \\
\cline { 2 - 6 } & Poz-57816 & Aragüés 5 & 1685 & 30 & 1596 \\
\cline { 2 - 6 } & Poz-57817 & Aragués 16 & 1790 & 30 & 1714 \\
\cline { 2 - 6 } & Liv-169822 & Lizara A 50 & 2415 & 30 & 2467 \\
\hline
\end{tabular}

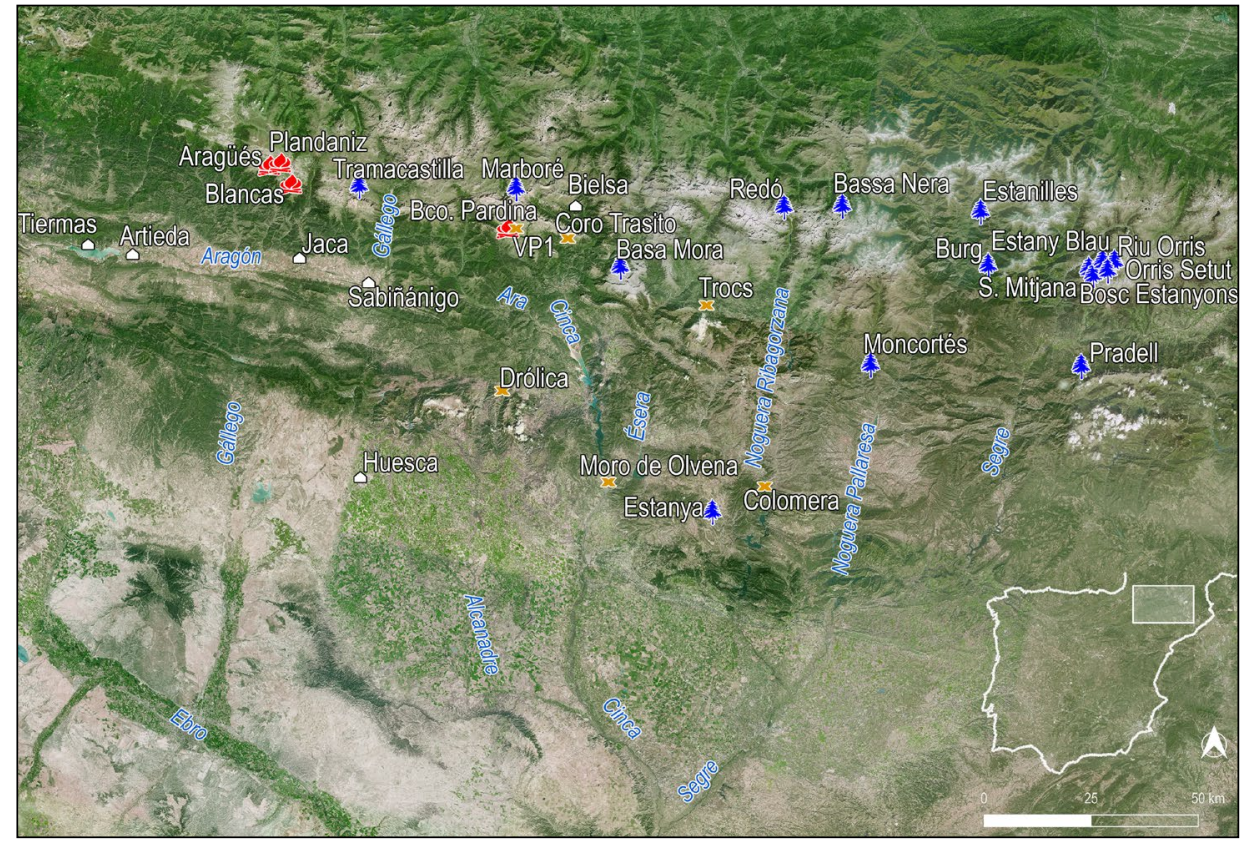

Figura 1. Mapa de localización con secuencias palinológicas lacustres y/o turbosas (símbolos azules), yacimientos arqueológicos (símbolos naranjas) y localidades con paleoincendios datados (símbolos rojos) considerados en este trabajo. 


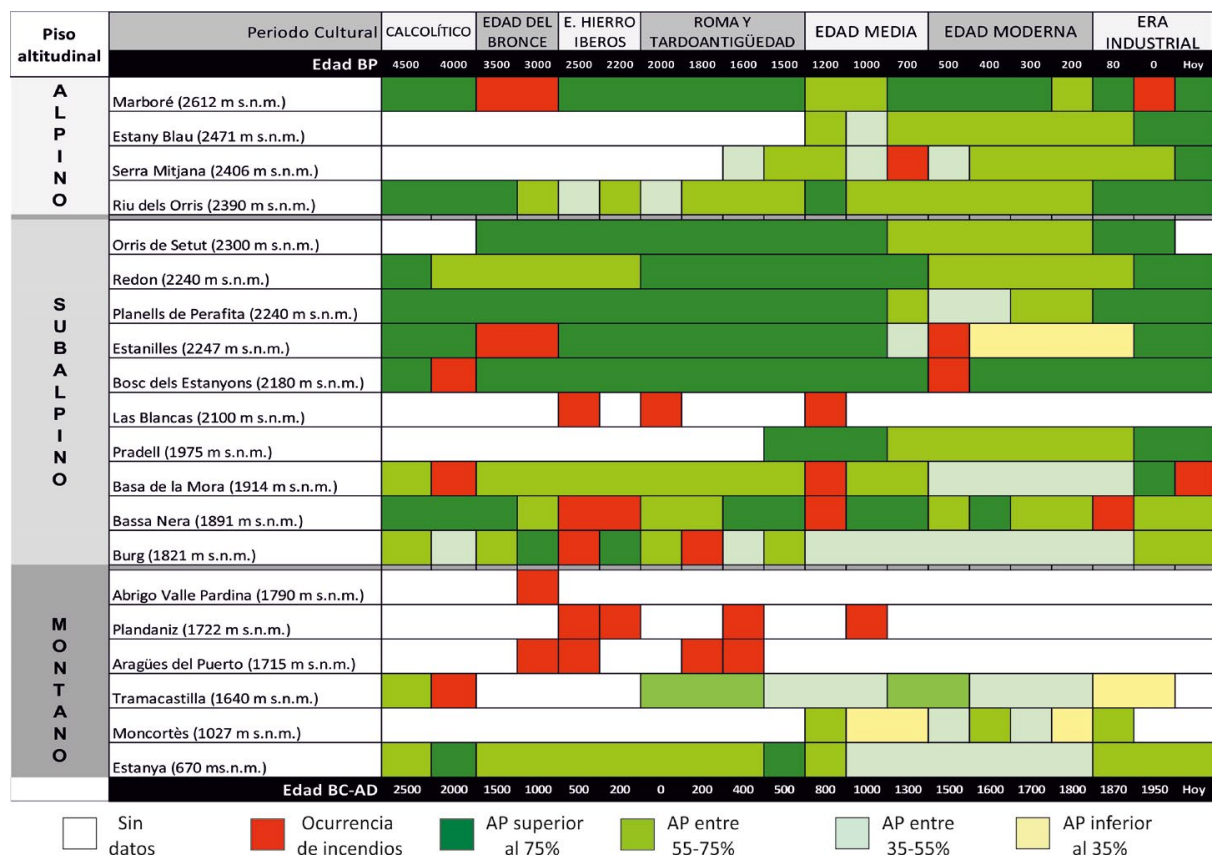

Figura 2. Comparación esquemática de niveles de paleoincendios y secuencias palinológicas pirenaicas del Holoceno Reciente localizadas a diferentes altitudes, representando intervalos con las proporciones medias de polen arbóreo y arbustivo $(A P)$ que registran, así como los episodios y/o periodos temporales en los que se han identificado picos de microcarbones y, por lo tanto, ocurrencia de incendios. Nótese la identificación de periodos culturales y sus límites cronológicos aproximados en la parte superior de la figura, así como las edades expresadas en años antes del presente (BP), $y$ en años antes y después de Cristo (BC-AD) en la inferior. La distribución de fechas no es uniforme ni está representada en escala. Las secuencias completas pueden consultarse en sus respectivas publicaciones: Marboré (Leunda et al., 2017); Estany Blau (Ejarque, 2009); Serra Mitjana (Miras et al., 2015); Riu dels Orris y Orris de Setut (Ejarque et al., 2010); Redon (Pla y Catalán, 2005); Planells de Perafita (Ejarque, 2009); Estanilles (Pérez-Obiol et al., 2012); Bosc dels Estanyons

(Miras et al., 2007); Pradell (Ejarque et al., 2009); Basa de la Mora (Pérez-Sanz et al., 2013);

Bassa Nera (Garcés-Pastor et al., 2017); Burg (Pèlachs et al., 2007); Tramacastilla (Montserrat, 1992); Moncortés (Rull et al., 2011); Estanya (González-Sampériz et al., 2017). Los niveles de paleoincendios localizados en depósitos de ladera (Aragüés del Puerto, Plandaniz, Abrigo Valle Pardina y Las Blancas) no van acompañados de datos palinológicos, por lo que aparecen en blanco excepto durante los periodos de ocurrencia de fuego (señalados en rojo).

Durante todo el Holoceno Reciente, las proporciones arbóreo-arbustivas (AP) registradas en las secuencias palinológicas del piso subalpino muestran valores que podemos considerar muy elevados, ya que mayoritariamente superan el $75 \%$ del espectro polínico, y en raras ocasiones bajan del 50\% (Fig. 2). Por su parte, los picos de microcarbones de estas secuencias, que se asocian con la ocurrencia de incendios, tampoco son continuos en el tiempo, ni periódicos, y aunque se deducen periodos de mayor concentración, como ocurre en torno a las Edades del Bronce y Hierro entre los 1700 y $2200 \mathrm{~m}$ de altitud (Fig. 2), no puede deducirse la intencionalidad de los mismos para mantener espacios abiertos a 
gran escala como se ha planteado en otras áreas montañosas de Iberia como la Cordillera Cantábrica, siempre en cotas altitudinales más bajas (Carracedo et al., 2018).

En este sentido, es importante destacar que los cambios que experimentan los bosques tras los incendios los hacen menos resistentes a nuevas quemas, independientemente de su origen, dado que cambia la estructura del sotobosque, la humedad relativa del combustible y por tanto la flamabilidad del mismo. Esto es lo que se conoce como "trampa de fuego" o fire trap de su definición en inglés, que es el fenómeno por el cual un sistema es más proclive a quemas tras eventos de incendios previos, hasta que, o bien cambian las condiciones climáticas que desencadenan el fuego o el combustible cambia en cantidad, estructura o composición debido a quemas sucesivas (Mouillot et al., 2003, Karavani et al., 2018, Santana et al., 2018). En definitiva, la vulnerabilidad de los bosques montanos o subalpinos al fuego aumenta con eventos de fuego iniciales, sin que la mano del hombre sea necesariamente la que los promueve.

Localmente no pueden descartarse acciones y/o estrategias antropogénicas (Ejarque et al., 2010; Bal et al., 2011), al contrario, ya que incluso existen yacimientos arqueológicos que presentan niveles de fumiers (Fig. 3) delatando su uso como rediles (Clemente et al., 2016). Los fumiers son depósitos que surgen con el Neolítico antiguo, ligados a la innovadora gestión ganadera que implica el uso de grandes cavidades (por primera vez o reocupando olvidadas cuevas paleolíticas) como rediles de ganado (Alday et al., 2012; Montes y Alday, 2012). Se caracterizan por la sucesión/intercalación de capas de variada coloración (negruzcas, marrones, grisáceas, blancas) resultantes de la acumulación del estiércol de los animales, tierra y restos vegetales, que además de fermentar de forma natural, en ocasiones eran quemados para higienizar el recinto (Fig. 3).
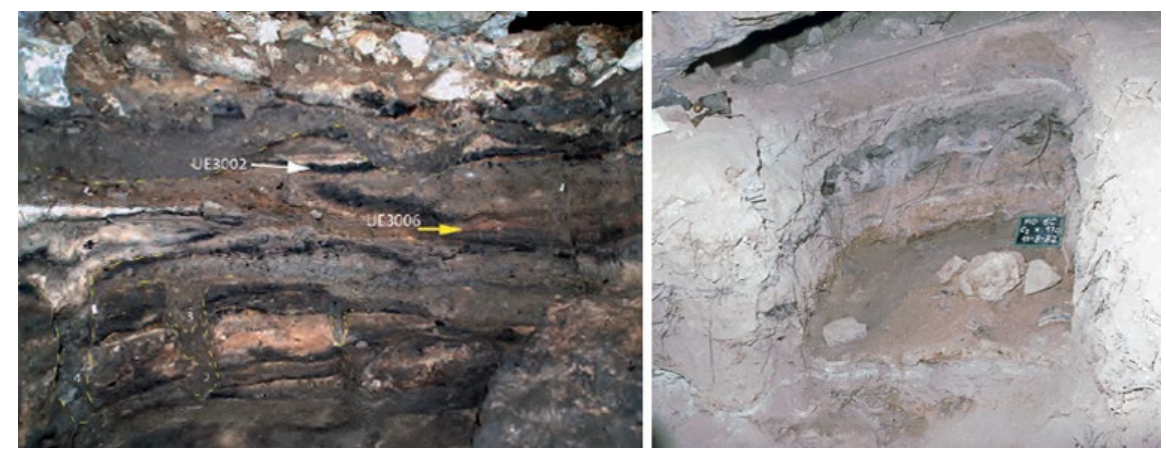

Figura 3. Niveles de fumier: neolíticos en la cueva de Coro Trasito (Tella-Sin, Huesca) a la izquierda (Clemente et al., 2014), y de la Edad del Bronce en la cueva del Moro de Olvena (Huesca) a la derecha.

En el entorno pirenaico se han descrito fumiers datados en el Neolítico antiguo en Coro Trasito (Clemente et al., 2014), Trocs (Rojo et al., 2013) y Colomera (Oms et al., 2013) (Fig. 1). Además, pueden intuirse en Chaves (Montes et al., 2016a) y Puyascada, a partir de la descripción que ofrece Baldellou (1987) de sus niveles neolíticos. Tras lo que parece el abandono de esta práctica en los milenios siguientes, los niveles de fumiers reaparecen a partir del Calcolítico (hace 4500 años) y perduran en el Bronce antiguo-medio en sitios como 
cueva Drólica (Montes et al., 2016b), quizás también en Moro de Olvena (Fig. 3, derecha), e incluso sobre rediles neolíticos, como en Coro Trasito (Gassiot et al., 2018).

Si hay rediles, hay ganado, y si hay ganado, necesariamente ha de haber pasto, pero estos niveles de fumiers también aparecen en cotas altitudinales variadas, al igual que ocurre con los niveles de paleoincendios (Figs. 2 y 4). Además, aunque la relación entre las cuevas-redil y el desarrollo de la primera ganadería es indudable, aún queda por explicar su carácter intermitente, y si el uso intencionado del fuego en el piso subalpino fue una práctica generalizada -o no- a escala regional en esa época.

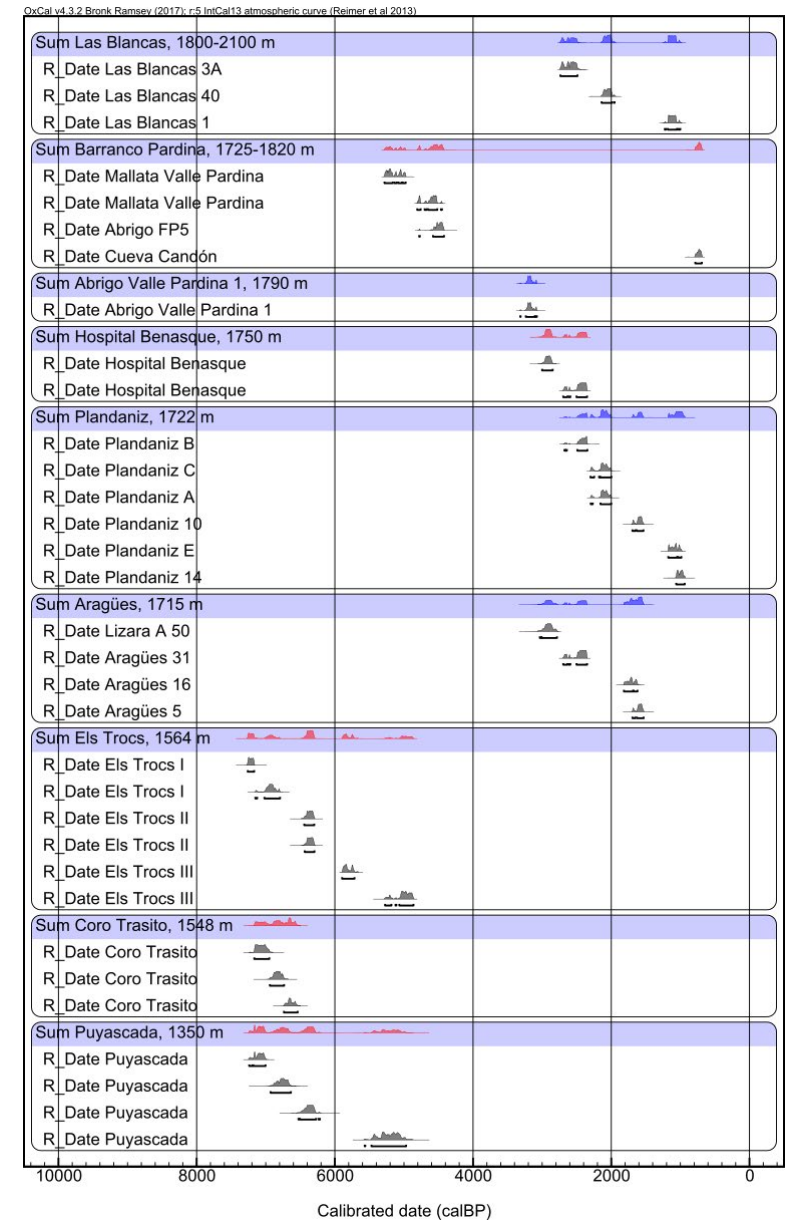

Figura 4. Distribución y suma de probabilidades del conjunto de dataciones procedentes de yacimientos arqueológicos (en rojo, con y sin niveles de fumier) y paleoincendios (en azul) del Pirineo Central desde hace 8000 años. Yacimientos: Hospital de Benasque (n=2) (Ona y Calastrenc, 2009), Barranco Pardina $(n=4)$ (Laborda et al., 2017), Coro Trasito, con fumiers ( $n=3)$ (Clemente et al., 2016), Puyascada ( $n=4)$ (Baldellou, 1987) y Trocs, con fumiers ( $n=6)$ (Rojo et al., 2013). Paleoincendios: Plandaniz ( $n=6)$, Las Blancas ( $(n=3)$, Aragüés $(n=4)$ y Valle Pardina $(n=1)$. El análisis ha sido realizado con OxCal v. 4.3

(Bronk Ramsey, 2009). La representación de los datos sigue el orden altitudinal. 
Secuencias largas de microcarbones del Pirineo Central obtenidas a partir de registros lacustres como los de la Basa de la Mora (BSM) y el Ibón de Marboré (MAR) (Fig. 1), también nos hablan de la dinámica del fuego, pero a escala más regional y continua en el tiempo que los niveles de paleoincendios mencionados (Fig. 5). En torno a 4000 años cal BP, en BSM (1900 m s.n.m.) se observa una mayor actividad del fuego que, sin embargo, no se corresponde con lo registrado en MAR (2600 m s.n.m.), que muestra un pico más tardío datado a comienzos de la Edad del Bronce (ca. 3500 años cal BP). En consonancia con BSM, también se registran picos de microcarbones/ incendios en cotas más bajas (Ibón de Tramacastilla, 1640 m s.n.m.), y más al este (Bosc dels Estanyons, Andorra, 2180 m s.n.m.) (Fig. 2). Sin embargo, ninguno de los paleoincendios localizados hasta el momento en la región, a pesar de estar en cotas similares (Tabla 1, Figs. 2 y 4), coincide con esta cronología, sino que todos son posteriores y han sido datados entre hace ca. 3000 y 1000 años, justo cuando tanto la secuencia de MAR como la de BSM indican una relativamente baja actividad del fuego (Leunda et al., en revisión).

Por otra parte, si observamos las ocupaciones de yacimientos arqueológicos, con y sin fumiers, existe un vacío significativo desde 4000 años cal BP, a pesar de que a partir de entonces aumentan las evidencias de incendios (Figs. 2, 4 y 5). Sólo en el Hospital de Benasque (1760 m s.n.m.), hay registro de ocupación humana entre 2500-2000 años cal BP coincidiendo con algunos de los paleoincendios datados y picos de microcarbones en Bassa Nera (1890 m s.n.m.) y el Lago Burg (1820 m s.n.m.) (Figs. 1, 2 y 4).

Esta aparente contradicción entre los registros largos de BSM y MAR, los picos aislados de otras secuencias palinológicas no analizadas en continuo, los niveles de paleoincendios datados y los vacíos de ocupación humana, probablemente corresponde a un escenario complejo tipo mosaico y de uso intermitente, con ocurrencia puntual de incendios locales y esporádicos. Estos incendios pudieron ser naturales o provocados por el hombre, pero desde luego no parecen responder a una práctica establecida de manera generalizada con el fin de mantener deforestada toda o buena parte del piso subalpino desde el inicio del Holoceno Reciente, ni desde antes.

Debe tenerse en cuenta, además, que hasta la Edad Media el número de cabezas de ganado en los valles pirenaicos tuvo que ser necesariamente pequeño, destinado más al autoconsumo (producción de carne, leche, piel y lana), que a mercados de envergadura que fueron inexistentes hasta que se implantó definitivamente la trashumancia. Es cierto que algunos autores (Higgs, 1976; Blondel, 2006) sugieren que en la Zona Mediterránea existieron rutas de trashumancia desde el Neolítico, como prolongación de los ancestrales movimientos estacionales de los herbívoros. Sin embargo, no hay evidencias de tales movimientos de largo alcance en un territorio tan fragmentado territorialmente (Escalona Monje, 2001; Galán Domingo y Ruiz-Gálvez, 2001). La presencia de manufacturas líticas del Neolítico y la Edad del Bronce a lo largo de las vías trashumantes es tomada con mucha cautela también por Davidson (1980). Esos pequeños rebaños de las Edades del Bronce y del Hierro (e incluso anteriores), debieron practicar una trasterminancia desde los asentamientos en los fondos de valle hasta el piso subalpino a comienzos de verano, y desde el subalpino a los valles al 
inicio del otoño (Montes et al., 2016a; Laborda et al., 2017; García-Ruiz y Lasanta, 2018; Montes et al., en prensa).

Para ello, solo sería necesario abrir pequeños claros a partir de 1600 m s.n.m., o aprovechar los existentes de manera natural. En cualquiera de los dos casos, no quedaría registro en los espectros polínicos de las secuencias lacustres, tal como se observa en la Fig. 2, ya que su extensión sería reducida y, por tanto, su impacto ambiental, muy limitado.

Que hubo pastoreo en el piso subalpino antes del periodo Romano, queda probado también por la presencia de numerosos monumentos megalíticos (túmulos, dólmenes, cromlechs, algún menhir), dispersos en ambiente claramente pastoral (Andrés Rupérez, 1999). Aunque la función primaria de algunas de esas estructuras es funeraria, todas ellas poseen un claro carácter de apropiación o marcaje territorial: ningún grupo es enterrado en un lugar que no considera suyo (Montes et al., 2016a). Cierto es que tales monumentos suelen localizarse próximos a los lugares de paso más importantes, no muy alejados de las áreas más accesibles de pastoreo, mientras que desaparecen en los más alejados, probablemente porque estos últimos se deforestaron muy tardíamente.

Con este escenario, no es posible a día de hoy, confirmar que los paleoincendios datados e identificados en localizaciones como Plandaniz, Aragüés del Puerto, Valle Pardina o Las Blancas (Tabla 1), así como el aumento de microcarbones que se registra en las secuencias lacustres de Marboré, Basa de la Mora (Fig. 5), Estanilles, Bosc del Estanyons, Bassa Nera o Burg (Fig. 2) durante el Calcolítico final y/o la Edad del Bronce, fueran provocados por los humanos, máxime teniendo en cuenta que un $50 \%$ de los fuegos que se producen actualmente en el Pirineo, intensamente antropizado, siguen siendo totalmente naturales (Ganteaume et al., 2013, European Forest Fire Information System). No obstante, la existencia de ganadería es clara, así como de una cultura que pudo tener ya en cuenta el escalonamiento altitudinal de los recursos pastorales en montaña, tal como se indica en Collis et al. (2016). Por eso, a menudo se sugiere en la literatura la ocurrencia de quemas tempranas de pequeña entidad para favorecer el pastoreo en verano, tanto en el Pirineo como el Sistema Ibérico (García-Ruiz et al., 2015, 2016a, 2016b).

Arqueológicamente, se registra cierto descenso y/o cambio de usos de la montaña pirenaica en época romana (Magallón y Sillières, 2013; Olesti Vila, 2017), a pesar de que se considera un primer pulso importante de explotación minera a escala del Mediterráneo (Domergue, 1990; Galop y Jalut, 1994; Pèlachs et al., 2009; Antolinos et al., 2010; López-Merino et al., 2014), como también indican Fernández Mier y Quirós Castillo (2015) para el noroeste de la Península Ibérica. En este contexto, quizá se podría incluir el Pirineo Central y, por ejemplo, la explotación de las minas de Bielsa, aunque no existen evidencias documentales ni materiales que puedan confirmarlo, ni más indicador paleoambiental que el sugerente pico de plomo identificado geoquímicamente a partir de fluorescencia de rayos X en la secuencia sedimentaria de Marboré entre hace 2300 y 2000 años (Oliva-Urcia et al., 2018), que no obstante puede ser debido a contaminación global y no a una intervención próxima. 

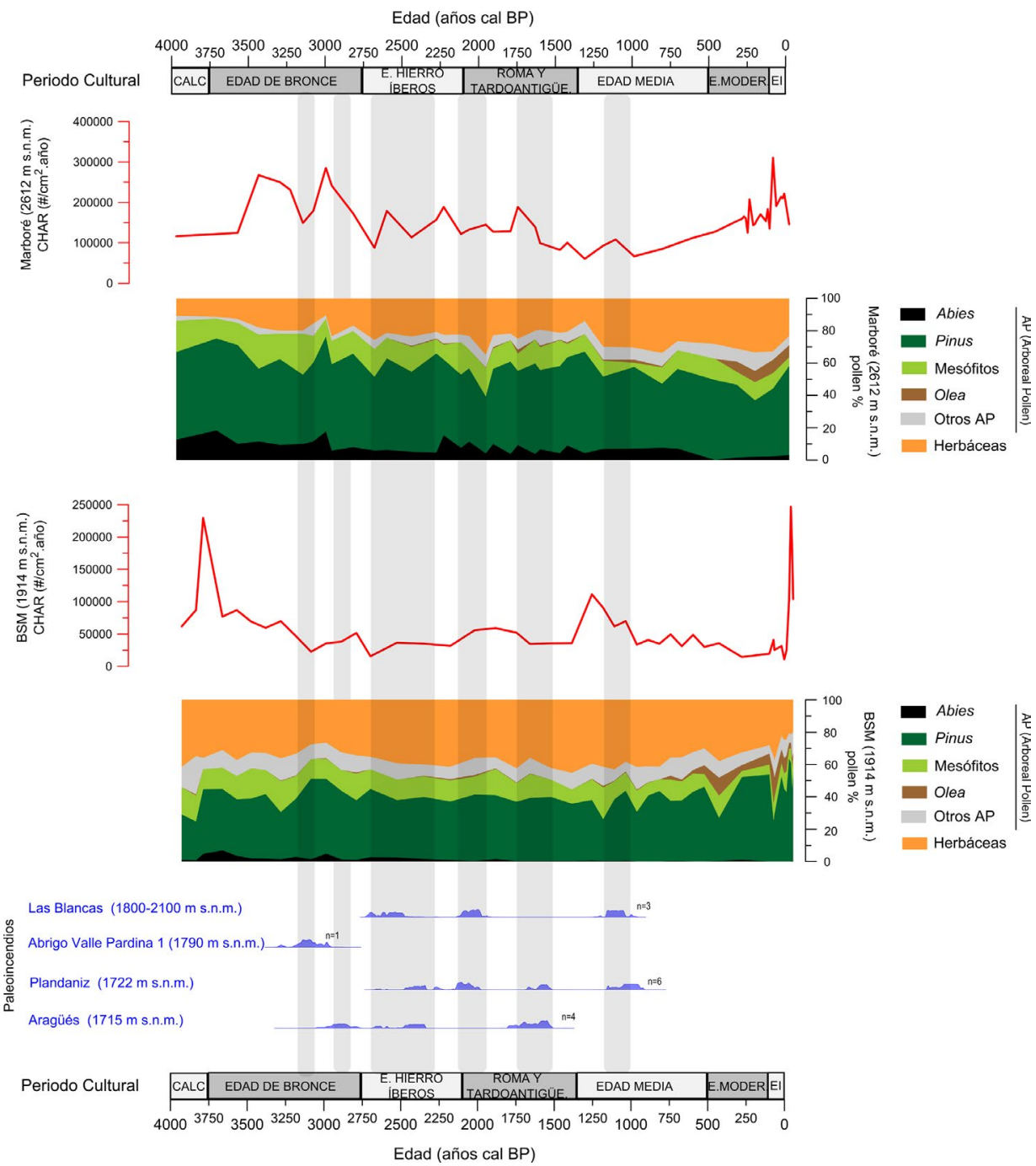

Figura 5. Secuencias de microcarbones (CHAR, en rojo) y principales taxones de los registros palinológicos de Marboré y Basa de la Mora (BSM), comparados con las curvas de distribución y suma de probabilidades del conjunto de paleoincendios de Las Blancas, Valle Pardina,

Plandániz y Aragüés, para los últimos 4000 años. Todos estos indicadores proceden del Pirineo Central. Los periodos culturales y sus límites cronológicos están también indicados junto a la cronología en años calibrados BP.

La mayoría de las minas metalíferas en los Pirineos son de pequeño tamaño, debido precisamente a su abundancia, que testimonia la fractura de los filones durante los procesos orogénicos. Algunas de ellas, como el plomo argentífero de Bielsa o de Géla se sitúan a más de $2000 \mathrm{~m}$ de altitud (las de Bielsa a unos 
2600 m s.n.m.), por lo que si su explotación en época moderna ha sido difícil y poco rentable, resulta complicado imaginarla en época ibérica y romana. La riqueza argentífera podría aplicarse mejor al conjunto de la Península y no específicamente a los Pirineos. Respecto al "Argentum oscense", Tito Livio utiliza esta expresión para referirse a las grandes cantidades de plata, acuñada o en lingotes, que era enviada a Roma tras los triunfos en Hispania de algunos generales (hay mención explícita a los años 195 y 179 a.C.). Ante las dudas que ha suscitado esa expresión, debido a la ausencia de correlación entre la importancia de la ceca de Bolskan (Osca, Huesca) y las supuestas cantidades masivas de moneda que llegaba a Roma, se han planteado varias explicaciones. Una, que Livio, escribiendo unos dos siglos después de los hechos narrados, cometiese un anacronismo y atribuyese a la ceca oscense, en funcionamiento en época augustea, acuñaciones muy anteriores. La segunda, que el término "argentum oscense" careciese de vinculación con la ciudad de Huesca. Villaronga (2004), incide al respecto en que la similitud de los alfabetos ibérico y osco (el pueblo prerromano de la península Itálica) provocase la confusión entre ambos al llegar a Roma moneda con textos escritos en escritura ibérica. No cabría, por tanto, buscar las fuentes minerales de las enormes cantidades de plata acuñadas específicamente en el Pirineo. Se trataría de monedas ibéricas de imitación griega, sí, pero procedentes de distintos lugares de la Península Ibérica: Emporion (Ampurias), principalmente, pero también otras ciudades importantes de la costa mediterránea, la más permeable a la romanización.

Es en torno a esas fechas de todos modos, cuando han sido datados algunos de los niveles de paleoincendios en el Pirineo Central (de nuevo sin constancia de si han sido naturales o producidos por el hombre) entre los 1600 y los $2100 \mathrm{~m}$ de altitud (Las Blancas 40 o incluso Plandaniz A y $\mathrm{C}$ algo antes: Tabla 1), pero las proporciones de AP en torno al cambio de era y la Tardoantigüedad, no registran bruscos descensos (Fig. 2), ni aperturas de paisaje prolongadas en el tiempo, por lo que el factor climático pudo perfectamente ser el agente originario del fuego. Los valores asociados al paisaje forestal superan el 75\% en todas las secuencias por encima de 1900-2000 m de altitud, excepto en la de Riu dels Orris en Andorra (Fig. 2); y en cotas inferiores, en ningún caso se registran proporciones por debajo del $55 \%$, reflejando un inequívoco paisaje aún muy forestado.

De época Medieval y Moderna existen documentos que confirman que el recurso económico fundamental en el Pirineo eran los pastos de alta montaña (Esteban-Amat, 2003; Fillat et al., 2008; Pascua Echegaray, 2012), y es en torno a hace 1000-1200 años, cuando vuelven a evidenciarse numerosos niveles de paleoincendios en cotas elevadas del Pirineo Central (ej., Las Blancas 1 y Plandániz E: Tabla 1), y picos de microcarbones en secuencias palinológicas como las de la Basa de la Mora y Bassa Nera (Fig. 2), aunque las proporciones de AP no descienden de forma generalizada ni en todas las cotas hasta después del 1300 AD (Fig. 2).

Ciertamente, en la historiografía medieval hay escasez de menciones a bosques, pero este hecho no implica per se la existencia de un paisaje totalmente deforestado desde hace milenios, sino quizá, un menor interés económico en su explotación por 
parte de las poblaciones montañesas (Utrilla Utrilla et al., 2003). La escala humana tiende a confundirse con la geológica, y la "memoria medieval/moderna" ha podido fácilmente extrapolar un modelo reciente de uso del territorio, mucho más atrás en el tiempo de lo que le corresponde. De hecho, en el siglo XI aún se menciona la existencia de bosques frondosos (silvas) en documentos aragoneses del rey Sancho Ramírez, que en los dos siglos siguientes, entonces sí, serían eliminados para la obtención de tierras para el pasto de ganado (Fillat et al., 2008; Pascua Echegaray, 2012).

Así, pues, parece cada vez más cierto que los grandes movimientos trashumantes en la Península Ibérica se establecieron a partir de la Reconquista, cuando los ganaderos de entonces (algunos de ellos convertidos en grandes ganaderos por privilegios reales), pudieron desplazarse anualmente a los pastos de invierno o extremos, una vez que los diferentes reinos alcanzaron cierta entidad territorial. Por ejemplo, Bielza de Ory et al. (1986) relacionan la evolución de la trashumancia con la consolidación del Reino de Aragón, de manera que las principales rutas trashumantes se establecieron entre 1170 y 1230. Hay otro hecho que sugiere la creciente importancia de la ganadería después de la Reconquista y su modesto papel anterior: la Reconquista supuso la reorganización de muchos territorios pastorales en, por ejemplo, las cabeceras de los valles de Hecho, Aragüés y Aísa, que pasaron a ser de dominio del Valle de Ansó, o las concesiones de pastos de verano en la cabecera del Valle del Río Aragón a localidades de fuera del valle (Jaca y Araguás del Solano) (Balcells, 1985), o los derechos cedidos a la Casa de Ganaderos de Zaragoza para pastar en los valles occidentales del Pirineo aragonés (Utrilla Utrilla et al., 2005). Es indudable que, si la actividad ganadera hubiera sido muy fuerte antes de la Reconquista, no habría sido posible tal redistribución y reorganización de los derechos de pastos en el alto Pirineo (García-Ruiz y Lasanta, 2018).

Es a partir del 1300 AD, cuando las proporciones arbóreo-arbustivas (AP) de todas las secuencias palinológicas pirenaicas del piso subalpino, ahora sí a escala regional, muestran un patrón común e inequívocamente claro: valores más bajos de AP y apertura de paisaje permanente en el tiempo (Fig. 2), aspecto que también se detecta en el relativamente próximo Lago de Arreo (Álava) (Corella et al., 2013) y de las ocupaciones pastoriles en las sierras de Aizkorri y Aralar (Fernández Mier y Quirós Castillo, 2015). Además, es en este periodo cuando se registra en todas ellas un significativo aumento de herbáceas nitrófilas tradicionalmente asociadas a actividades humanas, y porcentajes destacados de especies cultivadas como el cáñamo, el olivo o incluso la vid, además de los cereales (Riera et al., 2004; Morellón et al., 2011; Rull et al., 2011; González-Sampériz et al., 2017), reflejando el cultivo tradicional que impulsaron los monasterios en el piso montano esencialmente (Pascua Echegaray, 2012). Y es que el impacto de las primeras actividades agrícolas en el Pirineo Central se remonta a las Edades del Bronce y del Hierro (González-Sampériz et al., 2017), se mantiene con variaciones durante el periodo Ibero-Romano, y va en aumento en época Medieval y Moderna, registrándose incluso en secuencias elevadas como MAR y BSM en el caso del olivo (Fig. 5), por la fácil dispersión de su polen en altitud (Cañellas-Boltà et 
al., 2009). Parece evidente, además, que la ocupación cada vez más compleja del territorio en época bajomedieval mediante el aprovechamiento de los diferentes pisos geoecológicos (incluyendo no sólo los fondos de valle sino también, de forma generalizada, los pastos del piso subalpino) exigió prácticas de carácter colectivo o semi-colectivo, como también sugieren Fernández Mier y Quirós Castillo (2015) en otros sectores del norte de la Península.

No obstante, falta aún mucha información acerca de la construcción del paisaje agrario que pueda darnos pistas sobre el momento en que las actividades agrícolas representaron una profunda transformación estructural. De hecho, según Leveau y Martínez (2010), la agricultura pirenaica en época romana, debido a sus limitaciones ambientales, no ofrecía ninguna perspectiva para la exportación, por lo que no resultaba demasiado interesante para los autores romanos, que apenas la mencionan. El sistema de explotación habitual basado en villas rurales, fue poco frecuente (las mejor conocidas se sitúan en la Canal de Berdún, desde Artieda y Tiermas, hasta el entorno de Sabiñánigo), si bien es cierto que en las pocas localizadas es habitual encontrar instalaciones para el procesado de vino y aceite.

¿De cuándo son entonces los pueblos cuya agricultura se basa casi exclusivamente en el cultivo de terrazas? Es evidente que, en tales casos, la creación de los pueblos debió ser paralela a la de los campos aterrazados, aunque lamentablemente no sabemos cuándo se construyeron las primeras terrazas. Algunos autores aluden a la Edad del Bronce como periodo de inicio del aterrazamiento en la región Mediterránea (Asins, 2006), y estudios arqueológicos fechan terrazas de cultivo en Cataluña entre los siglos XIII y XIX (Kinnaird et al., 2017; Turner et al., 2017), es decir, desde la Baja Edad Media hasta época muy reciente. Más antiguos son los registros de terrazas en Salvatierra (Álava) con edades entre los siglos VI y XIII (Quirós Castillo, 2011), pero es posible que haya ejemplos más antiguos. Otros autores sin embargo, afirman que gran parte de las terrazas son de los siglos XVIII y XIX (Gómez Urdáñez y Moreno Fernández, 1997; Grove y Rackham, 2001), coincidiendo con el momento de mayor expansión demográfica y con la crisis de actividades artesanales. En todo caso, lo que rodea a la construcción de terrazas y su impacto en la vegetación y la erosión del suelo forma parte, por el momento, de una nebulosa científica. Por ejemplo, en el Pirineo aragonés los bancales sólo se utilizan al este del Valle del Gállego, mientras que al oeste predominan los campos en pendiente y los de agricultura nómada (Balcells, 1976; Lasanta et al., 1994). GarcíaRuiz y Lasanta (2018) sugieren que al oeste del Gállego las estructuras agrarias eran más provisionales y que la expansión de la agricultura era una solución ante la presión demográfica, mientras que hacia el este los campos aterrazados indican una cultura más permanentemente agrícola.

En cualquier caso, desde la Edad Media y hasta el inicio de la Era Industrial, la deforestación se registra a escala regional en todas las secuencias palinológicas (Fig. 2), con valores entre el 55 y el 35\% de AP (o incluso menos en Montcortés, por ejemplo), en la mayoría de secuencias por debajo de los 1900 m de altitud. En cotas más elevadas, las proporciones son mayores, pero siempre inferiores a las pre-existentes hasta el año 
1000 AD (Fig. 2), registrándose incluso, episodios severos de deforestación como el de Estanilles en época Moderna.

Este escenario de ritmo acelerado, coincidió con el crecimiento demográfico, que en general, alcanzó en el Pirineo Central sus cifras más elevadas a mediados del siglo XIX. La consecuencia más destacada fue la necesidad de ampliar la superficie cultivada hacia laderas más pendientes y alejadas, donde se invertía menos trabajo en conservación y los riesgos erosivos eran mayores (Lasanta, 1989; Lasanta et al., 2006). Además, los siglos XVIII y XIX coincidieron también con la crisis de muchas actividades artesanales, en especial la producción textil que presentaba una notable dispersión espacial, lo que obligaría, como sucedió en la montaña riojana (Gómez Urdáñez y Moreno Fernández, 1997), a forzar los mecanismos de subsistencia mediante la apertura de nuevos campos de cultivo. En el valle del río Ijuez, por ejemplo, la deforestación llegó hasta el siglo XIX, desencadenando intensos procesos de erosión (Sanjuán et al., 2016). De ahí que el delta del Ebro (que podría ser considerado como un excelente indicador del impacto humano y, por lo tanto, una expresión inequívoca del Antropoceno), experimentase una gran expansión espacial durante los siglos XVIII y XIX, si bien su crecimiento se anuncia claramente desde época Tardomedieval (Maldonado, 1972).

La situación actual nos enfrenta pues a un nuevo escenario (García et al., 2016). En cualquier caso, incluso hoy en día observamos cambios a los que no resulta fácil atribuir un origen exclusivamente antropogénico (Fig. 6), ya que el cambio global actual resulta, en la mayoría de los casos, de la combinación de un intenso incremento de temperaturas y del abandono de usos del suelo tradicionales (García-Ruiz et al., 2015). Sin duda, esa combinación de agentes de cambio tan intensamente inter-relacionados, es lo que define al Antropoceno como tal.

\section{Evidencias de intervención humana en el registro fósil: Conclusiones}

A lo largo de este trabajo se ha expuesto una reflexión acerca de las supuestas evidencias que señalan la deforestación del piso subalpino en el Pirineo a partir de intervención humana, recopilando información del registro palinológico, secuencias de microcarbones, niveles datados de paleoincendios e información arqueológica y documental. Con todo ello, se ha constatado la diversidad temporal existente entre todos los indicios, desde el Neolítico final-Calcolítico-Edad del Bronce hasta la Baja Edad Media, cuando ya sí que se registra un escenario de uso humano generalizable a escala regional y persistente en el tiempo.

El comienzo de las deforestaciones humanas buscando la expansión de prados de verano para el ganado y la conformación de lo que hoy denominamos paisajes culturales, varía según interpretación de autores. Pero si consideramos sensu stricto los valores que ofrecen los datos en su conjunto, el consenso es irrefutable: no ocurre a escala regional hasta la Baja Edad Media (últimos 700 años, 1300 AD). 


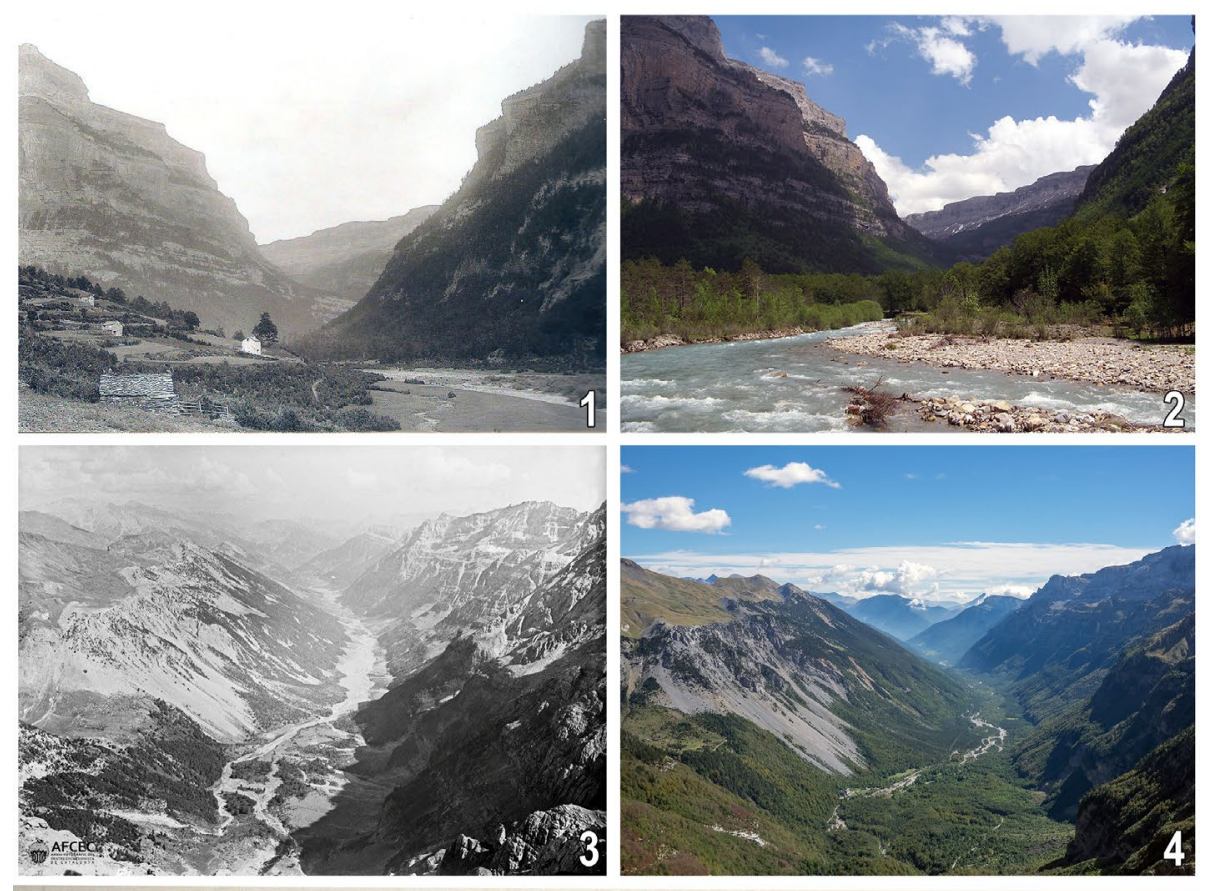

Figura 6. Imágenes comparativas del pasado y actualidad del Pirineo Central: 1. Valle de Ordesa en 1911, según L. Briet. 2. Valle de Ordesa en la actualidad. 3. Valle de Pineta en 1908 (Centre Excursionista de Catalunya). 4. Valle de Pineta en la actualidad. 5. Cañón de Añisclo en 1875, según F. Schrader. 
En cuanto al uso del fuego para este menester, las secuencias nos hablan de un incremento en la frecuencia de incendios a escala global desde finales del Holoceno Medio y comienzo del Reciente (esto es, en torno a hace unos 4000 años en algunas secuencias y alrededor de hace unos 3500 en otras), pero sin continuidad en el tiempo clara, ni desde luego periodicidad evidente. Al menos en el registro fósil disponible en la actualidad en la zona de estudio, por lo que los datos conocidos hasta hoy, evidencian fuegos que podrían ser, sin ningún problema, tanto antropogénicos como de origen natural, y siempre con carácter local y ocasional. Las posibles deforestaciones asociadas, especialmente en las Edades del Bronce y del Hierro, debieron tener por objeto atender a las necesidades de la población local, que practicaría una trasterminancia de corto recorrido desde los asentamientos del fondo del valle a los pastos subalpinos, donde hay evidencias de presencia humana estacional, desde el Calcolítico - Edad del Bronce e incluso antes.

Por último, las evidencias arqueológicas que buscan la demostración irrefutable del establecimiento de una actividad económica basada en la ganadería trashumante extensiva desde tiempos inmemoriales (incluso desde el Holoceno Temprano), son escasas y aisladas, y quizá herederas de un modelo posterior que, a partir de textos documentales, también se sitúa cronológicamente en el Medievo, pero no antes. No existe una confirmación clara de la ocurrencia de movimientos trashumantes de largo recorrido anteriores a la Reconquista, lo cual es lógico teniendo en cuenta que los mercados laneros nacionales e internacionales de envergadura sólo se consolidan a partir del siglo XIII.

¿Qué podemos considerar entonces como Antropoceno si lo identificamos con el origen de los paisajes culturales heredados de nuestro pasado tradicional? Según las evidencias actuales, con seguridad únicamente los últimos siglos, especialmente desde los siglos XII-XIII por lo que respecta a la deforestación general del piso subalpino, y desde el siglo XVII en el piso montano, como consecuencia del crecimiento demográfico y de la necesidad de ampliar la superficie cultivada hasta laderas pendientes y alejadas. El crecimiento del delta del Ebro desde la Edad Media y, muy particularmente, en los siglos XVIII y XIX, es quizás la mejor prueba de un impacto humano consistente y duradero en la cubierta vegetal y en los usos del suelo, con indudables repercusiones en el funcionamiento hidrológico y la erosión del suelo. Un aspecto clave y aún desconocido, es el momento en que se produce la construcción de terrazas en el Pirineo Central y sus efectos erosivos e hidrológicos, la mayoría de las cuales deben remontarse al momento en que se instalan los asentamientos cuya agricultura, ha dependido siempre y fundamentalmente de los campos abancalados. Ir más atrás en el tiempo para localizar su origen, o al contrario, acercarlo hasta sólo unas pocas generaciones o décadas, dependerá sin duda, del devenir de las investigaciones en los próximos años.

\section{Agradecimientos}

Este trabajo se ha llevado a cabo con el apoyo de los proyectos DINAMO 3 (CGL2015-69160R), DINAMO2 (CGL2012-33063), ESPAS (CGL2015-655698-R), 
CLIP (HAR2014-59042-P) y GAS (HAR2017-85023-P), financiados por el Ministerio de Economía y Competitividad. R. Domingo es investigador Ramón y Cajal (RyC201312613) y M. Leunda investigadora en formación del programa FPI del MINECO (FPI ref.: BES-2013-063753). Un agradecimiento especial va dirigido a María Ruiz del Árbol Moro, investigadora del Instituto de Historia-CSIC, por sus sugerencias bibliográficas sobre minería romana; y a Elena Royo y Raquel López Cantero, ayudantes de investigación del IPE-CSIC, por el tratamiento de laboratorio de las muestras palinológicas de las secuencias paleoambientales de MAR y BSM. El equipo firmante de este trabajo pertenece a los grupos de investigación reconocidos del Gobierno de Aragón "Procesos Geoambientales y Cambio Global" (E02_17R) y "Primeros Pobladores del Valle del Ebro-PPVE" (H14_17R).

\section{Referencias}

Aitchinson, J. 1995. Cultural landscape in Europe: a geographical perspective. In: B. von Droste, H. Plachter, M. Rössler (Eds.), Cultural landscapes of universal value. Gustav Fischer Verlag, Jena, Stuttgart, New York, pp. 272-288.

Alday, A., Montes, L., Baldellou, V. 2012. El Neolítico en la Cuenca del Ebro. En: M. Rojo, R. Garrido, I. García (Coords.), El Neolítico en la Península Ibérica y su contexto europeo. Ediciones Cátedra, Madrid,pp. 291-331.

Andrés Rupérez, T. 1999: Los caminos y los sepulcros megalíticos. En: M.A. Magallón (Coord.), Caminos y comunicaciones en Aragón. Fernando el Católico. Zaragoza: 29-42.

Antolinos, J.A., Fabre J.M., Rico, C. 2010. Las minas romanas de Carthago Noua. Avance de las investigaciones en la Rambla del Abenque (Sierra de Cartagena). Mastia 9, 151-177. https:// dialnet.unirioja.es/descarga/articulo/4261336.pdf.

Antrop, M. 2005. Why landscapes of the past are important for the future. Landscape and Urban Planning 70, 21-34. https://doi.org/10.1016/j.landurbplan.2003.10.002.

Aranbarri, J., González-Sampériz, P., Iriarte, E., Moreno, A., Rojo-Guerra, M., Peña-Chocarro, L., Valero-Garcés, B., Leunda, M., García-Prieto, E., Sevilla-Callejo, M., Gil-Romera, G., Magri, D., Rodríguez-Lázaro, J. 2015. Human-landscape interactions in the ConquezuelaAmbrona Valley (Soria, continental Iberia): from the early Neolithic land use to the origin of the current oak woodland. Palaeogeography, Palaeoclimatology, Palaeoecology 436, 41-57. https://doi.org/10.1016/j.palaeo.2015.06.030.

Arias Maldonado, M. 2018. Antropoceno. La política en la era humana. Ed. Taurus, 256 pp.

Asins, S. 2006. Linking historical Mediterranean terraces with water catchment, harvesting and distribution structures. En: J.P. Morel (Ed.), The archaeology of crop fields and gardens. Ediplugia, Bari, pp. 21-40.

Bal, M.C., Pèlachs, A., Perez-Obiol, R., Julià, R., Cunill, R. 2011. Fire history and human activities during the last 3300 cal y BP in Spain's Central Pyrenees: the case of the Estany de Burg. Palaeogeography, Palaeoclimatology, Palaeoecology 300, 179-190. https://doi. org/10.1016/j.palaeo.2010.12.023.

Balcells, E. 1976. El Pirineo: Contraste de paisajes; enlace de pueblos. Imprenta del Ministerio de Asuntos Exteriores, Madrid.

Balcells, E. 1985. Valle del Aragón de Canfranc (Estudio descriptivo de recursos e historia de su utilización). Pirineos 125, 117-140.

Baldellou, V. 1987. Avance al Estudio de la Espluga de la Puyascada. Bolskan 4, 3-41. http:// revistas.iea.es/index.php/BLK/article/view/861/858. 
Barreiro-Lostres, F., Moreno, A., González-Sampériz, P., Giralt, S., Nadal-Romero, E., ValeroGarcés B. 2017. Erosion in Mediterranean mountain landscapes during the last millennium: a quantitative approach based on lake sediment sequences (Iberian Range, Spain). Catena 149, 782-798. https://doi.org/10.1016/j.catena.2016.05.024.

Bernabeu, J., García Puchol, O., Orozco-Köhler, T. 2018. New insights relating to the beginning of the Neolithic in the eastern Spain: Evaluating empirical data and modelled predictions. Quaternary International 470, 439-450. https://doi.org/10.1016/j.quaint.2017.03.071.

Bielza de Ory, V., Corral Lafuente, J.L., Escolano Utrilla, S., Laliena Corbera, C., Sesma Muñoz, A., Ubieto Arteta, A. 1986. Estudio histórico-geográfico del valle de Bielsa (Huesca). Instituto de Estudios Altoaragoneses, Huesca, 224 pp.

Blondel, J. 2006. The "design" of Mediterranean landscapes: A millennial story of humans and ecological systems during the historic period. Human Ecology 34, 713-729. https://doi. org/10.1007/s10745-006-9030-4.

Bronk Ramsey, C. 2009. Bayesian analysis of radiocarbon dates. Radiocarbon 51 (1), 337-360. https://doi.org/10.1017/S0033822200033865.

Cañellas-Boltà, N., Rull, V., Vigo, J., Mercadé, A. 2009. Modern pollen-vegetation relationships along an altitudinal transect in the central Pyrenees (southwestern Europe). The Holocene 19, 1185-1200. https://doi.org/10.1177/0959683609345082.

Carracedo, V., Cunill, R., García-Codron, J.C., Pèlachs, A., Pérez-Obiol, R., Soriano, J.M., 2018. History of fires and vegetation since the Neolithic in the Cantabrian Mountains (Spain). Land Degradation Development 29, 2060-2072. https://doi.org/10.1002/ldr.2891.

Carrión, J.S., Fernández, S., González-Sampériz, P., Gil-Romera, G., Badal, E., Carrión- Marco, Y., López-Merino, L., López-Sáez, J.A., Fierro, E., Burjachs, F. 2010. Expected trends and surprises in the Lateglacial and Holocene vegetation history of the Iberian Peninsula and Balearic Islands. Review of Palaeobotany and Palynology 162, 458-475. https://doi. org/10.1016/j.revpalbo.2009.12.007.

Catalán, J., Pèlachs, A., Gassiot, E., Antolín, F., Ballestero, A., Batalla, M., Burjachs, F., Buchaca, T., Camarero, L., Clement, I., Clop, X., García, D., Giralt, S., Lluch, L., Madella, M., Mazzuco, N., Mur, E., Ninyerola, M., Obea, L., Oltra, J., Pérez-Obiol, R., Piqué, R., Pla-Rabés, S., Rondón, C.R., Rodríguez, J.M., Rodriguez, D., Sáez, A., Soriano, J.M. 2013. Interacción entre clima y ocupación humana en la configuración del paisaje vegetal del Parque Nacional de Aigüestores i Estany de Sant Maurici a lo largo de los últimos 15.000 años. In: L. Ramírez, B. Asensio (Eds.), Proyectos de Investigación en Parques Nacionales: 2009-2012. Organismo Autónomo Parques Nacionales, Madrid, pp. 71-92.

Clemente, I., Gassiot, E., Rey, J., Mazzucco, N., Obea, L. 2014. Cort o Transito -Coro Trasito- o corral de tránsito: una cueva pastoril del Neolítico antiguo en el corazón de Sobrarbe. En: I. Clemente-Conte, E. Gassiot Ballbè, J. Rey Lanaspa (Eds.), Sobrarbe Antes de Sobrarbe. Pinceladas de Historia de Los Pirineos. Centro de Estudios de Sobrarbe, pp. 7-32.

Clemente, I., Gassiot, E., Rey, J., Antolín, F., Obea, L., Viñerta, A,. Saña Seguí. M. 2016. Coro Trasito (Tella-Sin, HU) un asentamiento de pastores en el Pirineo Central con dataciones del neolítico antiguo y del Bronce medio. En: L. Lorenzo Lizalde. J.M. Rodanés Vicente (Eds.), Actas I Congreso de Arqueología y Patrimonio Aragonés, Zaragoza, Colegio Oficial de Doctores y Licenciados en Filosofía y Letras y Ciencias de Aragón, pp. 75-84.

Collis, J., Pearce, M., Nicolis, F. (Eds.) 2016. Summer farms. Seasonal exploitation of the uplands from Prehistory to the present. J.R. Collis Publications, Sheffield Archaeological Monographs, 16. ISBN 9780906090558.

Corella, J.P., Stefanova, V., El Amjoumi, A., Rico, E., Giralt, S., Moreno, A., Plata-Moreno, A., Valero-Garcés,B.L.2013.A2500-yearmulti-proxy reconstruction of climatechange andhuman 
activities in northern Spain: The Lake Arreo record. Palaeogeography, Palaeoclimatology, Palaeoecology 386, 555-568. https://doi.org/10.1016/j.palaeo.2013.06.022.

Davidson, I. 1980. Transhumance, Spain and Ethnoarchaeology. Antiquity 54, 144-147. https:// doi.org/10.1017/S0003598X00103035.

Domergue, C. 1990. Les Mines de la Péninsule Ibérique dans l'Antiquité romaine. Collection de l’École Française de Rome, Paris, 625 pp.

Ejarque, A. 2009. Génesis y configuración micro-regional de un paisaje cultural pirenaico de alta montaña durante el Holoceno: estudio polínico y de otros indicadores paleoambientales en el valle del Madriu-Perafita-Claror (Andorra). Tesis Doctoral, Institut Català d'Arqueologia Clàssica. Universitat Rovira i Virgili, Tarragona, 363 pp.

Ejarque, A., Julià, R., Riera, S., Palet, J.M., Orengo, H.A., Miras, Y., Cascón, C. 2009. Tracing the history of highland human management in the Eastern Pre-Pyrenees: an interdisciplinary palaeoenvironmental study at the Pradell fen. Spain. The Holocene 19 (8), 1241-1255. https:// doi.org/10.1177/0959683609345084.

Ejarque, A., Miras, Y., Riera, A., Palet, J.M., Orengo, H.A. 2010. Testing microregional variability in the Holocene shaping of high mountain cultural landscapes: a palaeoenvironmental casestudy in the eastern Pyrenees. Journal of Archaeological Science 37, 1468-1479. https://doi. org/10.1016/j.jas.2010.01.007.

Escalona Monge, J. 2001. Jerarquización social y organización del espacio. Bosques y pastizales en la Sierra de Burgos (siglos X-XII). En: J. Gómez-Pantoja (Ed.), Los rebaños de Gerión. Pastores y trashumancia en Iberia antigua y medieval. Casa de Velázquez, Madrid, pp. 109137.

Esteban Amat, A. 2003. La humanización de las altas cuencas de la Garona y las Nogueras $(4500 a C-1995 d C$ ). Serie Histórica Naturaleza y Parques Nacionales, Ministerio de Medioambiente, $469 \mathrm{pp}$.

Fernández Mier, M., Aparicio Martínez, P., González Álvarez, D., Fernández-Fernández, J., Alonso González, P. 2013. La formación de los paisajes agrarios del Noroeste peninsular durante la Edad media (siglos V al XII). Debates de Arqueología Medieval 3, 359-374. https://eprints.ucm.es/24474/1/Art_dam_pro.pdf.

Fernández Mier, M., Quirós Castillo, J.A. 2015. El aprovechamiento de los espacios comunales en el noroeste de la Península Ibérica entre el periodo romano y el medieval. Il Capitale Culturale 12, 689-717. http://doi.org/10.13138/2039-2362/1196.

Fillat, F., García-González, R., Gómez, D., Reiné, R. 2008. Pastos del Pirineo. Consejo Superior de Investigaciones Científicas, Premios Félix de Azara, Diputación de Huesca. Madrid, 319 pp.

Finney, S.C., Edwards, L.E. 2016. The "Anthropocene" epoch: Scientific decision or political statement? GSA Today 3, 4-10. https://doi.org/10.1130/GSATG270A.1.

Galán Domingo, E., Ruiz-Gálvez, M. 2001. Rutas ganaderas, trasterminancia y caminos antiguos. El caso del Occidente peninsular entre el Calcolítico y la Edad del Hierro. En: J. GómezPantoja (Ed.), Los rebaños de Gerión. Pastores y trashumancia en Iberia antigua y medieval. Casa de Velázquez, Madrid, pp. 263-278.

Galop, D. 1999. La forêt, l'homme et le troupeau dans les Pyrénées. 6000 ans d'histoire de l'environnement entre Garonne et Méditerranée. GEODE, Toulouse.

Galop, D., Jalut, G. 1994. Differential human impact and vegetation history in two adjacent Pyrenean valleys in the Ariège basin, southern France, from 3000 BP to the present. Vegetation History and Archaeobotany 3, 225-244. https://doi.org/10.1007/BF00195199.

Galop, D. 2006. La conquête de la montagne pyrénéenne au Néolithique. Chronologie, rythmes et transformations des paysages à partir des données polliniques. En: J. Guilaine (Dir.) Populations néolithiques et environnement. Paris, Errance, pp. 279-295. 
Galop,D., Ruis, D., Cugny, C., Mazier, F. 2013. A history of long-term human-environment interactions in the French Pyrenees inferred from the pollen data. In: L.R. Lozny (Ed.), Continuity and change in cultural adaptation to mountain environments. Studies in human ecology and adaptation. Springer, New York, pp. 19-30. https://doi.org/10.1007/978-1-4614-5702-2_3.

Ganteaume, A., Camia, A., Jappiot, M., San-Miguel-Ayanz, J., Long-Fournel, M., Lampin, C. 2013. A review of the main driving factors of forest fire ignition over Europe. Environmental Management 51, 651-662. https://doi.org/10.1007/s00267-012-9961-z.

Garcés-Pastor, S., Cañellas-Boltà, N., Pèlachs, A., Soriano, J.-M., Pérez-Obiol, R., Pérez-Haase, A., Calero, M.-A., Andreu, O., Escolà, N., Vegas-Vilarrúbia, T. 2017. Environmental history and vegetation dynamics in response to climate variations and human pressure during the Holocene in Bassa Nera, Central Pyrenees. Palaeogeography, Palaeoclimatology, Palaeoecology 479, 48-60. https://doi.org/10.1016/j.palaeo.2017.04.016.

García, M.B., Alados, C.L., Antor, R., Benito Alonso, J.L., Camarero, J.J., Carmena, F., Errea, P., Fillat, F., García-González, R., García-Ruiz, J.M., Gartzia, M., Gomez-García, D., Gómez, I., González-Sampériz, P., Gutiérrez, E., Jiménez, J.J., López-Moreno, J.I., Mata, M.P., Moreno, A., Montserrat, P., Nuche, P., Pardo, I., Revuelto, J., Rieradevall, M., Sáiz, H., Tejero, P., Vicente-Serrano, S.M., Villagrasa, E., Villar, L., Valero-Garces, B.L. 2016. Integrando escalas y métodos LTER para comprender la dinámica global de un espacio protegido de montaña: el Parque Nacional de Ordesa y Monte Perdido. Ecosistemas 25 (1), 19-30. https://doi.org/10.7818/ECOS.2016.25-1.04.

García-Ruiz, J.M., López-Moreno, J.I., Lasanta, T., Vicente-Serrano, S., González-Sampériz, P., Valero-Garcés, B.L., Sanjuán, Y., Beguería, S., Nadal-Romero, E., Lana-Renault, N., Gómez-Villar, A. 2015. Los efectos geoecológicos del Cambio Global en el Pirineo Central español: una revisión a distintas escalas espaciales y temporales. Pirineos 170, e012. http:doi. org/10.1989/Pirineos.2015.170005.

García-Ruiz, J.M., Sanjuán, Y., Gil-Romera, G., González-Sampériz, P., Beguería, S., Arnáez, J., Coba-Pérez, P., Gómez-Villar, A., Álvarez-Martínez, J., Lana-Renault, N., Pérez-Cardiel, E., López de Calle, C. 2016a. Mid and late Holocene forest fires and deforestation in the subalpine belt of the Iberian Range, northern Spain. Journal of Mountain Science 13 (10), 1760-1772. https://doi.org/10.1007/s11629-015-3763-8.

García-Ruiz, J.M., Sanjuán, Y., Arnáez, J., Beguería, S., Gómez-Villar, A., Álvarez-Martínez, J., Lana-Renault, N., Coba-Pérez, P. 2016b. La evolución del piso subalpino en la Sierra de Urbión (Sistema Ibérico, Norte de España): Un modelo de impacto geoecológico de actividades humanas en el Valle de Ormazal. Pirineos 171, e022. https://doi.org/10.3989/ Pirineos.2016.171006.

García-Ruiz, J.M., Lasanta, T. 2018. El Pirineo aragonés como paisaje cultural. Pirineos 137, e038. https://doi.org/10.3989/pirineos.2018.173005.

Gassiot, E. 2016. Montañas humanizadas. Arqueología del pastoralismo en el Parque Nacional d'Aigüestortes i Estany de Sant Maurici, Madrid, Organismo Autónomo de Parques Nacionales.

Gassiot, E., Rodríguez-Antón, D., Burjachs, F., Antolín, F., Ballesteros, A. 2012. Poblamiento, explotación y entorno natural de los estadios alpinos y subalpinos del Pirineo central durante la primera mitad del Holoceno. Cuaternario y Geomorfología 26, 29-45. https://recyt.fecyt. es/index.php/CUGEO/article/view/19412.

Gassiot, E., Rey, J., Clemente, I., Obea, L., Díaz, S., Quesada, M., García, V., García, D., Rodríguez, D. 2018. Estructuras de almacenaje prehistóricas en la cueva redil de Coro Trasito (TellaSin, Huesca). En: L. Lorenzo Lizalde, J.M. Rodanés Vicente (Eds.), Actas II Congreso de Arqueología y Patrimonio Aragonés, Zaragoza, Colegio Oficial de Doctores y Licenciados en Filosofía y Letras y Ciencias de Aragón, p. 41-48. 
Gil-Romera, G., Carrión, J. S., Pausas, J. G., Sevilla-Callejo, M., Lamb, H. F., Fernández, S., Burjachs, F. 2010. Holocene fire activity and vegetation response in SouthEastern Iberia. Quaternary Science Reviews 29, 1082-1092. https://doi.org/10.1016/j. quascirev.2010.01.006.

Gil-Romera, G., González-Sampériz, P., Lasheras-Álvarez, L., Sevilla-Callejo, M., Moreno, A., Valero-Garcés, B., López-Merino, L., Carrión, J.S., Pérez Sanz, A., Aranbarri, J., García Prieto Fronce, E. 2014. Biomass-modulated fire dynamics during the last glacialinterglacial transition at the central Pyrenees (Spain). Palaeogeography, Palaeoclimatology, Palaeoecology 402, 113-124. https://doi.org/10.1016/j.palaeo.2014.03.015.

Gómez Urdáñez, J.L., Moreno Fernández, J.R. 1997. El problema agrario en las sierras de La Rioja: de la propiedad a la subsistencia (siglos XVIII y XIX). Agricultura y Sociedad 82, 79-113. https://www.mapa.gob.es/ministerio/pags/biblioteca/revistas/pdf_ays/a082_03.pdf.

González-Sampériz, P., Aranbarri, J., Pérez-Sanz, A., Gil-Romera, G., Moreno, A., Leunda, M., Sevilla-Callejo, M., Corella, J.P., Morellón, M., Oliva, B., Valero-Garcés, B. 2017. Environmental and climate change in the southern Central Pyrenees since the last glacial maximum: a view from the lake records. Catena 149, 668-688. https://doi.org/10.1016/j. catena.2016.07.041.

Grove, D., Rackham, O. 2001. The nature of Mediterranean Europe: An ecological history. Yale University Press, New Haven.

Higgs, E.S. 1976. The history of European agriculture: The uplands. Philosophical Transactions of the Royal Society B 275, 159-173. https://doi.org/10.1098/rstb.1976.0078.

Juan-Cabanilles, J., Martí-Oliver, B. 2002. Poblamiento y procesos culturales en la Península Ibérica del VII milenio a.C. (8000-5500 B.P.). Una cartografía de la neolitización. Saguntum Extra 5, 45-87.

Karavani, A., Boer, M.M., Baudena, M., Colinas, C., Díaz-Sierra, R., Pemán, J., Luis, M. de, Enríquez-de-Salamanca, Á., de Dios, V.R. 2018 Fire-induced deforestation in droughtprone Mediterranean forests: drivers and unknowns from leaves to communities. Ecological Monographs 88, 141-169. https://doi.org/10.1002/ecm.1285.

Kinnaird, T., Bolòs, J., Turner, A., Turner, S. 2017. Optically-stimulated luminiscence profiling and dating of historic agricultural terraces in Catalonia (Spain). Journal of Archaeological Science 78, 66-77. https://doi.org/10.1016/j.jas.2016.11.003.

Laborda, R., Villalba-Mouco, V., Lanau, P., Gisbert, M., Sebastián, M., Domingo, R., Montes, L. 2017. El Puerto Bajo de Góriz (Parque Nacional de Ordesa y Monte Perdido). Ocupación y explotación de un paisaje de alta montaña desde la prehistoria hasta el siglo XX. Bolskan 26, 9-30. https://zaguan.unizar.es/record/70729? ln=es.

Lasanta, T. 1989. Evolución reciente de la agricultura de montaña: el Pirineo aragonés. Geoforma Ediciones, 220 pp., Logroño.

Lasanta, T., Ortigosa Izquierdo, L.M., García-Ruiz, J.M. 1994. Distribución espacial de diferentes modelos de campos de cultivo en el Pirineo Central español. Ería 33, 63-72. https://doi. org/10.17811/er.0.1994.63-72.

Lasanta, T., Beguería, S., García-Ruiz, J.M. 2006. Geomorphic and hydrological effects of traditional shifting agriculture in a Mediterranean mountain area, Central Spanish Pyrenees. Mountain Research and Development 26 (2), 146-152. https://doi.org/10.1659/02764741(2006)26[146:GAHEOT]2.0.CO;2.

Leunda, M., González-Sampériz, P., Gil-Romera, G., Aranbarri, J., Moreno, A., Oliva-Urcia, B., Sevilla-Callejo, M., Valero-Garces, B. 2017. The Late-Glacial and Holocene Marbore Lake sequence (2612 m a.s.l., Central Pyrenees, Spain): testing high altitude sites sensitivity to millennial scale vegetation and climate variability. Global and Planetary Change 157, 214231. https://doi.org/10.1016/j.gloplacha.2017.08.008. 
Leunda M., Gil-Romera, G., Daniau, A.L., González-Sampériz, P. (en revisión). Holocene fire-vegetation dynamics in the Central Pyrenees (Spain). Catena (SI Women in Geoecology).

Leveau, P., Martínez, J.M.P. 2010. Les Pyrénées romaines, la frontière, la ville et la montagne. L'apport de l'archéologie du paysage. Pallas 82, 171-198. https://www.jstor.org/ stable/43596908?seq=1\#page_scan_tab_contents.

López-Merino, L., Cortizas, A.M., López-Sáez, J.A. 2010. Early agriculture and palaeoenvironmental history in the North of the Iberian Peninsula: a multiproxy analysis of the Monte Areo mire (Asturias, Spain). Journal of Archaeological Science 37, 1978-1988. https://doi.org/10.1016/j.jas.2010.03.003.

López-Merino, L., Martínez Cortizas, A., Reher, G.S., López-Sáez, J.A., Mighall, T.M., Bindler, R. 2014. Reconstructing the impact of human activities in a NW Iberian Roman mining landscape for the last 2500 years. Journal of Archaeological Science 50, 208-218. https://doi. org/10.1016/j.jas.2014.07.016.

López-Sáez, J.A., Blanco-González, A., López-Merino, L., Ruiz-Zapata, M.B., Dorado, M., Pérez-Díaz, S., Valdeolmillos, A., Burjachs, F. 2009. Landscape and climatic changes during the end of the Late Prehistory in the Amblés Valley (Ávila, central Spain), from 1200 to 400 cal BC. Quaternary International 200, 90-101. https://doi.org/10.1016/j. quaint.2008.07.010.

Magallón, M.A., Sillières, P. 2013. Labitolosa (La puebla de Castro, province de Huesca, Espagne). Une cité romaine de l'Hispanie citérieure. Ausonius Éditions, Mémoires 33. Bordeaux, 499 pp.

Maldonado, A. 1972. El delta del Ebro, estudio sedimentario y estratigráfico. Boletín de Estratigrafía 1, 1-486.

Malm, A., Hornborg, A. 2014. The geology of mankind? A critique of the Anthropocene narrative. The Anthropocene Review 1 (1), 62-69. https://doi.org/10.1177/2053019613516291.

Martí-Oliver, B., Juan-Cabanilles, J. 2014. Los primeros agricultores neolíticos mediterráneos (VI-V milenios a.C.). Protohistoria de la Península Ibérica. Del Neolítico a la romanización, 19-41 Universidad de Burgos/Fundación Atapuerca.

Martínez de Pisón, E. 2009. Miradas sobre el paisaje. Biblioteca Nueva, Madrid, 285 pp.

Miras, Y., Ejarque, A., Riera, S., Palet, J.M., Orengo, H., Euba, I. 2007. Dynamique holocene de la vegetation et occupation des Pyrenees andorranes depuis le Neolithique ancien, d'apres l'analyse pollinique de la tourbiere de Bosc dels Estanyons (2180 m, Vall del Madrid, Andorra). Comptes Rendus Palevol 6, 291-300. https://doi.org/10.1016/j.crpv.2007.02.005.

Miras, Y., Ejarque, A., Riera Mora, S., Orengo, H. A., Palet Martinez, J. M. 2015. Andorran high Pyrenees (Perafita Valley, Andorra): Serra Mijtana fen. Grana 54(4), 313-316. https://doi.or $\mathrm{g} / 10.1080 / 00173134.2015 .1087590$.

Monastersky, R. 2015. Anthropocene: the human age. Nature 519, 144-147. https://doi. org/10.1038/519144a.

Montes, L. Alday, A. 2012. Enredados en la malla neolítica de la cuenca del río Ebro. Redes, continuidades y cambios. Rubricatum 5, 51-60. https://core.ac.uk/download/ pdf/39122466.pdf.

Montes, L., Domingo Martínez, R., Sebastián López, M., Lanau Hernáez, P. 2016a. Construyendo un paisaje. Megalitos, arte esquemático y cabañeras en el Pirineo central. ARPI. Arqueología y Prehistoria del Interior Peninsular 4, 248-263. https://zaguan.unizar.es/record/63392/files/ texto_completo.pdf.

Montes, L., Bea-Martínez, M., Domingo, R., Sánchez, P., Alcolea, M., Sebastián, M. $2016 b$. La gestión prehistórica de un territorio en la montaña Prepirenaica: Tierra Bucho (Huesca, España). Munibe. Antropología y Arqueología 67, 349-382. http://www.aranzadi.eus/ fileadmin/docs/Munibe/maa.2016.67.mis07.pdf. 
Montes, L., Domingo, R., Laborda, R., Lanau, P., Villalba-Mouco, V., Gisbert, M., Sebastián, M. (en prensa). Le canyon de la Pardina et ses estives. Approche archéologique d'un territoire de haute montagne dans le Parc National d'Ordesa et du Mont Perdu (Huesca). En: M. Deschamps, S. Costamagno, P.Y. Milcent, J.M. Pétillon, C. Renard, N. Valdeyron (Dirs.), Actes du 142e Congrès national des sociétés historiques et scientifiques «Circulations montagnardes, circulations européennes», Pau, 24-29 avril 2017, Paris, Editions de CTHS.

Montserrat, J. 1992. Evolución glaciar y postglaciar del clima y la vegetación en la vertiente sur del Pirineo: Estudio palinológico. Instituto Pirenaico de Ecología, 147 pp., Zaragoza.

Morellón, M., Valero-Garcés, B., González-Sampériz, P., Vegas-Villarrúbia, T., Rubio, E., Rieradevall, M., Delgado-Huertas, A., Mata, P., Romero, Ó., Engstrom, D., López-Vicente, M., Navas, A., Soto, J. 2011. Climate changes and human activities recorded in the sediments of Lake Estanya (NE Spain) during the Medieval Warm Period and Little Ice Age. Journal of Paleolimnology 46, 423-452. https://doi.org/10.1007/s10933-009-9346-3.

Mouillot, F., Ratte, J.-P., Joffre, R., Mouillot, D., Rambal, S. 2005. Long-term forest dynamic after land abandonment in a fire prone Mediterranean landscape (central Corsica, France). Landscape Ecology 20, 101-112. https://doi.org/10.1007/s10980-004-1297-5.

Olesti Vila, O. 2017. Augusto y el control de los territorios pirenaicos. Gerión 35, 163 pp. http:// revistas.ucm.es/index.php/GERI/article/view/56143.

Oliva-Urcia, B., Moreno, A., Leunda, M., Valero-Garcés, B., González-Sampériz, P., Gil-Romera, G., Mata, P., HORDA group. 2018. Last deglaciation and Holocene environmental change at high altitude in the Pyrenees: the geochemical andpaleomagnetic record from Marboré Lake (N Spain). Journal of Paleolimnology 59, 349-371. https://doi.org/10.1007/s10933017-0013-9.

Oms, F.X., López-García, J.M., Mangado, X., Martín, P., Mendiela, S., Morales, J.I., Rodríguez, A., Rodríguez-Cintas, A., Yubero, M. 2013. Habitat en cova i espai pels ramats ca. 62006000 BP: dades preliminars de la Cova Colomera (Prepirineu de Lleida) durant el neolític antic. Saguntum 45, 25-38. https://ojs.uv.es/index.php/saguntum/article/view/2007/2772.

Ona, J.L., Calastrenc, C. 2009. Historia de los Hospitales de Benasque y Bañeras de Luchón (Ocho siglos de Hospitalidad al pie del Aneto). Fundación Hospital de Benasque.

Orengo, H.A., Palet, J.M., Ejarque, A., Miras, Y., Riera, S. 2014. Shifting occupation dynamics in the Madriu-Perafita-Claror valleys (Andorra) from the early Neolithic to the Chalcolithic: the onset of high mountain cultural landscapes. Quaternary International 353, 140-152. https://doi.org/10.1016/j.quaint.2014.01.035.

Pallaruelo, S. 1988. Pastores del Pirineo. Madrid, Ministerio de Cultura, D.L. Dirección general de Bellas Artes y Archivos. Madrid. 229 pp.

Pascua Echegaray, E. 2012. Señores del Paisaje. Ganadería y recursos naturales en Aragón, siglos XIII-XVII. Universitat de Valencia-PUV, 327 pp

Pèlachs, A., Soriano, J.M., Nadal, J., Esteban, A. 2007. Holocene environmental history and human impact in the Pyrenees. Contributions to Science 3, 421-429. https://www.researchgate.net/ publication/29495176_Holocene_enviromental_history_and_human_impact_in_the_Pyrenees.

Pèlachs, A., Nadal, J., Soriano, J.M., Molina, D., Cunill, R., 2009. Changes in Pyrenean woodlands as a result of the intensity of human exploitation: 2,000 years of metallurgy in Vallferrera, northeast Iberian Peninsula. Vegetation History and Archaeobotany 18, 403-416. https://doi. org/10.1007/s00334-009-0218-6.

Peña-Chocarro, L., Zapata, L., Iriarte, M.J., González Morales, M., Straus, L.G. 2005. The oldest agriculture in northern Atlantic Spain: new evidence from El Mirón Cave (Ramales de la Victoria, Cantabria). Journal of Archaeological Science 32, 579-587. https://doi. org/10.1016/j.jas.2004.12.001. 
Peña-Chocarro, L., Pérez-Jordà, G., Morales, J. 2018. Crops of the first farming communities in the Iberian Peninsula. Quaternary International 470, 369-382. https://doi.org/10.1016/j. quaint.2017.06.002.

Pérez-Díaz, S., Lopez-Sáez, J.A., Galop, D. 2015. Vegetation dynamics and human activity in the Western Pyrenean Region during the Holocene. Quaternary International 364, 65-77. https://doi.org/10.1016/j.quaint.2014.10.019.

Pérez-Díaz, S., López-Sáez, J. A., de la Fuente, S. N., Ruiz-Alonso, M. 2018. Early farmers, megalithic builders and the shaping of the cultural landscapes during the Holocene in Northern Iberian mountains. A palaeoenvironmental perspective. Journal of Archaeological Science: Reports 18, 463-474. https://doi.org/10.1016/j.jasrep.2018.01.043.

Pérez-Obiol, R., Bal, M.-C., Pèlachs, A., Cunill, R., Soriano, J.M. 2012. Vegetation dynamics and anthropogenically forced changes in the Estanilles peat bog (southern Pyrenees) during the last seven millennia. Vegetation History and Archaeobotany 21, 385-396. https://doi. org/10.1007/s00334-012-0351-5.

Pérez-Sanz, A., González-Sampériz, P., Valero-Garcés, B., Moreno, A., Morellón, M., Sancho, C., Belmonte, A., Gil-Romera, G., Sevilla, M., Navas, A. 2011. Clima y Actividades Humanas en la Dinámica de la Vegetación Durante los Últimos 2000 Años en el Pirineo Central: el Registro Palinológico de la Basa de la Mora (Macizo de Cotiella). Zubía 23, 17-38. https:// dialnet.unirioja.es/servlet/articulo?codigo $=3817943$.

Pérez-Sanz, A., González-Sampériz, P., Moreno, A., Valero-Garcés, B., Gil-Romera, G., Rieradevall, M., Tarrats, P., Lasheras-Álvarez, L., Morellón, M., Belmonte, A., Sancho, C., Sevilla-Callejo, M., Navas, A. 2013. Holocene climate variability, vegetation dynamics and fire regime in the central Pyrenees: the Basa de la Mora sequence (NE Spain). Quaternary Science Reviews 73, 149-169. https://doi.org/10.1016/j.quascirev.2013.05.010.

Pla, S., Catalán, J. 2005. Chrysophyte cysts from lake sediments reveal the submillennial winter/spring climate variability in the northwestern Mediterranean region throughout the Holocene. Climate Dynamics 24, 263-278. https://doi.org/10.1007/s00382-0040482-1.

Quirós Castillo, J.A. 2011. La formación de los paisajes medievales en el norte peninsular: agricultura y ganadería en los siglos V-XII. Debates de Arqueología Medieval 1, 161-165.

Rendu C. 2003. La montagne d'Enveig, un estive pyrénéenne dans la longue durée, Le canet en Roussillon, Trabucaire.

Rendu, C., Calastrenc, C., Le Couédic, M., Berdoy, A. 2016. Estives d'Ossau, 7000 ans de pastoralisme dans les Pyrénées, Toulouse, Le Pas d'Oiseau.

Revelles, J. 2017. Archaeology of Neolithisation. Human-environment interactions in the NE Iberian Peninsula during the Early Neolithic. Journal of Archaeological Science 15, 437-445. https://doi.org/10.1016/j.jasrep.2016.02.004.

Riera, S., Wansard, G., Julià, R. 2004. 2000-year environmental history of a karstic lake in the Mediterranean pre-Pyrenees: The Estanya lakes (Spain). Catena 55, 293-324. https://doi. org/10.1016/S0341-8162(03)00107-3.

Roberts, N., Fyfe, R.M., Woodbridge, J., Gaillard, M.J., Davis, B.A., Kaplan, J.O., Marquer, L., Mazier, F., Nielsen, A.B., Sugita, S., Trondman, A.-K., Leydet, M. 2018. Europe's lost forests: a pollen-based synthesis for the last 11,000 years. Scientific reports 8,716 . https:// doi.org/10.1038/s41598-017-18646-7.

Rojo, M., Garrido, R., García, I. 2012. El Neolítico en la Península Ibérica y su contexto europeo. Ediciones Cátedra, Madrid.

Rojo, M., Peña, L., Royo, J.I., Tejedor, C., García, I., Arcusa, H., Garrido, R., Moreno, M., Mazzuco, N., Gibaja, J.F., Ortega, D., Kromer, B., Alt, K. 2013. Pastores trashumantes del Neolítico antiguo en un entorno de alta montaña: secuencia crono-cultural de la Cova 
de Els Trocs (San Feliú de Veri, Huesca). Boletín del Seminario de Arte y Arqueología 79, 9-55.

Rull, V., González-Sampériz, P., Corella, J.P., Morellón, M., Giralt, S. 2011. Vegetation changes in the southern Pyrenean flank during the last millennium in relation to climate and human activities: the Montcortes lacustrine record. Journal of Paleolimnology 46, 387-404. https:// doi.org/10.1007/s10933-010-9444-2.

Rull, V. 2018. El Antropoceno. Colección ¿Qué sabemos de? Ed. Consejo Superior Investigaciones Científicas, Los libros de la catarata. Madrid, $141 \mathrm{pp}$.

Sanjuán, Y., Gómez-Villar, A., Nadal-Romero, E., Álvarez-Martínez, J., Arnáez, J., SerranoMuela, M.P., Rubiales, J.M., González-Sampériz, P., García-Ruiz, J.M. 2016. Linking land cover changes in the sub-alpine and montane belts to changes in a torrential river. Land Degradation and Development 27, 179-189. https://doi.org/10.1002/ldr.2294.

Santana, V.M., Baeza, M.J., Valdecantos, A., Vallejo, V.R. 2018. Redirecting fire-prone Mediterranean ecosystems toward more resilient and less flammable communities. Journal of Environmental Management 215, 108-115. https://doi.org/10.1016/j.jenvman.2018.03.063.

Turner, S., Bolòs, J., Kinnaird, T. 2017. Changes and continuities in a Mediterranean landscape: a new interdisciplinary approach to understanding historic character in western Catalonia. Landscape Research 43 (7), 1-17. https://doi.org/10.1080/01426397.2017.1386778.

Utrilla, P. 2002. Epipaleolíticos y neolíticos del Valle del Ebro. Saguntum Extra 5, 179-208. https://ojs.uv.es/index.php/saguntumextra/article/view/10700/9898.

Utrilla Utrilla, J.F., Laliena, C., Navarro, G. 2003. La evolución histórica del paisaje rural en los Pirineos durante la Edad Media: explotación agropecuaria y recursos forestales. En: A Sabio, I. Iriarte (Eds.), La construcción histórica del paisaje agrario en España y Cuba. Los Libros de la Catarata. Madrid, pp. 57-69.

Utrilla Utrilla, J.F., Laliena, C., Navarro, G. 2005. Los recursos naturales y su transformación en los Pirineos aragoneses durante la Edad Media. En: Les ressources naturelles des Pyrénées du Moyen Âge à l'époque modern. Actes du Congrès International RESOPYR-1, Presses Universitaires de Perpignan, pp. 19-48.

Vannière, B. 2001. Feu, agro-pastoralisme et dynamiques environnementales en France durant l'Holocène, analyse du signal incendie, approches sédimentologiques et études de cas en Berry, Pyrénées et Franche-Comté. Institut national agronomique, Paris-Grignon.

Villaronga, L. 2004. Numismàtica antiga de la Península Ibèrica: introducció al seu estudi. Institut d'Estudis Catalans, Barcelona, 333 p.

Walker, M.J.C., Berkelhammer, M., Björck, S., Cwynar, L.C., Fisher, D.A., Long, A.J., Lowe, J.J., Newnham, R.M., Rasmussen, S.O., Weiss, H. 2012. Formal subdivision of the Holocene Series/Epoch: a Discussion Paper by a Working Group of INTIMATE (Integration of icecore, marine and terrestrial records) and the Subcommission on Quaternary Stratigraphy (International Commission on Stratigraphy). Journal of Quaternary Science 27, 649-659. https://doi.org/10.1002/jqs.2565.

Waters, C.N., Zalasiewicz, J., Summerhayes, C., Fairchild, I.J., Rose, N.L., Loader, N.J., Shotyk, W., Cearreta, A., Head, M.J., Syvitski, J.P.M., Williams, M., Wagreich, M., Barnosky, A.D., An, Z., Leinfelder, R., Jeandel, C., Gałuszka, A., Ivar do Sul, J.A., Gradstein, F., Steffen, W., McNeill, J.R., Wing, S., Poirier, C., Edgeworth, M. 2018. Global Boundary Stratotype Section and Point (GSSP) for the Anthropocene Series: Where and how to look for potential candidates. Earth-Science Reviews 178, 379-429. https://doi.org/10.1016/j. earscirev.2017.12.016.

Zalasiewicz, J., Waters, C.N., Summerhayes, C.P., Wolfe, A.P., Barnosky, A.D., Cearreta, A., Crutzen, P., Ellis, E., Fairchild, I.J., Gałuszka, A., Haff, P., Hajdas, I., Head, M.J., Ivar do Sul, J.A., Jeandel, C., Leinfelder, R., McNeill, J.R., Neal, C., Odada, E., Oreskes, N., Steffen, 
W., Syvitski, J., Vidas, D. 2017. The Working Group on the Anthropocene: Summary of evidence and interim recommendations. Anthropocene 19, 55-60. https://doi.org/10.1016/j. ancene.2017.09.001.

Zapata, L., Peña-Chocarro, L., Pérez-Jordà, G., Stika, H.-P. 2004. Early Neolithic agriculture in the Iberian Peninsula. Journal of World Prehistory 18, 283-325. https://doi.org/10.1007/ s10963-004-5621-4. 